Draft VERsion September 20, 2016

Preprint typeset using LATEX style AASTeX6 v. 1.0

\title{
A DEEP X-RAY VIEW OF THE BARE AGN Ark 120. II. EVIDENCE FOR Fe K EMISSION TRANSIENTS
}

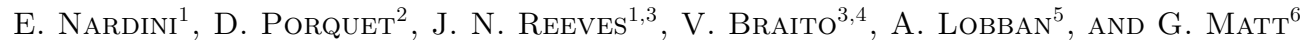 \\ ${ }^{1}$ Astrophysics Group, School of Physical and Geographical Sciences, Keele University, Keele, Staffordshire ST5 5BG, UK \\ ${ }^{2}$ Observatoire Astronomique de Strasbourg, CNRS, UMR 7550, 11 rue de l'Université, 67000 Strasbourg, France \\ ${ }^{3}$ Department of Physics, University of Maryland Baltimore County, 1000 Hilltop Circle, Baltimore, MD 21250, USA \\ ${ }^{4}$ INAF - Osservatorio Astronomico di Brera, via E. Bianchi 46, I-23807 Merate, Italy \\ ${ }^{5}$ Department of Physics and Astronomy, University of Leicester, Leicester LE1 7RH, UK \\ ${ }^{6}$ Dipartimento di Matematica e Fisica, Università degli Studi Roma Tre, Via della Vasca Navale 84, I-00146 Roma, Italy
}

\begin{abstract}
We report on the results from a large observational campaign on the bare Seyfert galaxy Ark 120, jointly carried out in 2014 with XMM-Newton, Chandra, and NuSTAR. The fortunate line of sight to this source, devoid of any significant absorbing material, provides an incomparably clean view to the nuclear regions of an active galaxy. Here we focus on the analysis of the iron fluorescence features, which form a composite emission pattern in the 6-7 keV band. The prominent $\mathrm{K} \alpha$ line from neutral iron at $6.4 \mathrm{keV}$ is resolved in the Chandra High-Energy Transmission Grating spectrum to a full-width at half maximum of $4700_{-1500}^{+2700} \mathrm{~km} \mathrm{~s}^{-1}$, consistent with an origin from the optical broad-line region. Excess components are detected on both sides of the narrow K $\alpha$ line: the red one $(6.0-6.3 \mathrm{keV})$ clearly varies in strength in about one year, and hints at the presence of a broad, mildly asymmetric line from the accretion disk; the blue one $(6.5-7.0 \mathrm{keV})$, instead, is likely a blend of different contributions, and appears to be constant when integrated over long enough exposures. However, the Fe K excess emission map computed over the 7.5 days of the XMM-Newton monitoring shows that both the red and the blue features are actually highly variable on timescales of $\sim 10-15$ hours, suggesting that they might arise from short-lived hotspots on the disk surface, located within a few tens of gravitational radii from the central supermassive black hole and possibly illuminated by magnetic reconnection events. Any alternative explanation would still require a highly dynamic, inhomogeneous disk/coronal system, involving clumpiness and/or instability.
\end{abstract}

Keywords: galaxies: active - galaxies: individual (Ark 120) - X-rays: galaxies.

\section{INTRODUCTION}

About four decades have passed since a crude shape of the X-ray continuum from active galactic nuclei (AGNs) was first revealed (e.g., Ives et al. 1976; Mushotzky et al. 1980), yet the origin of the main spectral components that have been progressively brought out is still largely unclear. The primary X-ray emission, usually described by a power law with high-energy ( $\gtrsim 100 \mathrm{keV}$ ) cutoff, is thought to stem from inverse Compton scattering of ultraviolet (UV) disk photons in a coronal region of hot electrons (e.g., Haardt \& Maraschi 1993). The nature of this corona and of its coupling with the disk, however, remains unknown. Neither do widely accepted explanations exist for the soft (Done et al. 2012; Vasudevan et al. 2014) or hard X-ray excesses (Risaliti et al. 2013; Miller \& Turner 2013), or the broad iron fluorescence lines at $\sim 5-7 \mathrm{keV}$ (Tanaka et al. 1995; Inoue \& Matsumoto 2003). In fact, intricate absorption by neutral and/or ionized gas along the line of sight can reproduce any sort of spectral curvature or deviation from the power-law continuum. The unique potential of X-ray observations as a means of probing the immediate surroundings of an accreting supermassive black hole (SMBH) is therefore best exploited in the so-called bare active galaxies, where any obscuration effect is negligible.

The nearby $(z \simeq 0.0327$; Osterbrock \& Phillips 1977) Seyfert galaxy Ark 120 is arguably the most remarkable object in the bare AGN subclass. It is the X-ray brightest source of this type $\left(f_{0.3-10 \mathrm{keV}} \sim f_{14-195 \mathrm{keV}} \sim 7 \times 10^{-11}\right.$ $\mathrm{erg} \mathrm{s}^{-1} \mathrm{~cm}^{-2}$; this work; Baumgartner et al. 2013), and it offers the cleanest view to the central engine. Early 
high-resolution data taken with the Reflection Grating Spectrometer (RGS) onboard XMM-Newton posed stringent constraints on the presence of any warm absorber, whose column density would be at least an order of magnitude lower than found in a typical Seyfert 1 (Vaughan et al. 2004). Moreover, no evidence for UV absorption emerges from the Hubble Space Telescope spectrum (Crenshaw et al. 1999). Since it is not affected by any significant foreground screen, the observed X-ray emission of Ark 120 can thus be regarded as representative of the intrinsic high-energy output of an AGN, and of the physical processes associated with the inner accretion flow, such as Comptonization and/or relativistic reflection. Ark 120 displays all the X-ray spectral traits expected for an unobscured, radiatively efficient $\mathrm{SMBH}$, namely a smooth soft excess below $2 \mathrm{keV}$, an iron K-shell line complex possibly including a broad and skewed component, and a Compton reflection hump peaking at about $30 \mathrm{keV}$ (e.g., Nardini et al. 2011). The soft excess has always been noticeable in Ark 120 since the first X-ray observations (Brandt et al. 1993), but its featureless appearance is compatible with several interpretations, the most relevant of which invoke either the Comptonization of the seed UV photons in the warm disk atmosphere, or the blurring of the soft X-ray reflection-line forest in the strong gravity regime. The broad iron line and the Compton hump are even more puzzling, since their relative intensity apparently decreased in a recent (2013), combined observation with XMM-Newton and NuSTAR, when the source was found to be two times fainter than usual (Matt et al. 2014).

In order to address these open issues and shed new light on the intrinsic X-ray emission of AGNs, Ark 120 was the target of an extensive campaign carried out in $2014 \mathrm{March}$, consisting of an XMM-Newton long look ( $650 \mathrm{ks}$, or 7.5 days) with joint high-resolution view of the iron-K band with the Chandra High-Energy Transmission Grating (PI: D. Porquet), plus a simultaneous NuSTAR observation. Here we focus on the properties of the iron K-shell emission features, which can be studied in great detail thanks to the unprecedented depth of the new data sets. The paper is organized as follows: in Section 2 we introduce the various observations and provide the basic information on the data reduction, while Section 3 concerns the analysis of the time-averaged spectra. Section 4 deals with the subsequent discovery of the short-term variability of iron fluorescence. These results and their possible implications on the physics of the X-ray corona are further discussed in Section 5, and conclusions are drawn in Section 6. The consequences of the soft X-ray, grating spectra on the bare character of Ark 120 are the subject of a companion paper (Reeves et al. 2016), while the broadband analysis of the XMM-Newton and NuSTAR observations, and the modeling of the optical to hard X-ray spectral energy distribution will be presented in a forthcoming work (D. Porquet et al. 2016, in preparation).

\section{OBSERVATIONS AND DATA REDUCTION}

Ark 120 was observed by XMM-Newton over four consecutive orbits between 2014 March 18-24 (Table 1). The event files were reprocessed with the Science Analysis System (SAS) v14.0, applying the latest calibrations available on 2015 February. Due to the source brightness, the EPIC instruments were operated in Small Window mode, although this course of action was not enough to prevent pile-up in the MOS cameras. Only the EPIC/pn (Strüder et al. 2001) data were therefore taken into account, selecting the event patterns 0-4 (single and double pixel). The source spectra and light curves were extracted from circular regions centered on the target, with radius of $30^{\prime \prime}$ to avoid the edge of the chip. The background was evaluated over areas of the field of view where the contamination from the bright source is minimal, and its count rate is non negligible only in the final parts of each orbit, which were discarded. After the correction for dead time and background flaring, the total net exposure was $\sim 330 \mathrm{ks}$. Redistribution matrices and ancillary response files for all the data sets were generated with the SAS tasks rmfgen and arfgen. Since there are several tens of counts per native energy channel in the $\mathrm{Fe} \mathrm{K}$ band (equivalent to a $\gg 5 \sigma$ significance at the observed background level), the 3-10 keV spectra were grouped into $30-\mathrm{eV}$ bins not to unduly oversample the actual EPIC/pn resolution. This delivers about 1100 and 300 counts per bin around 5 and $8 \mathrm{keV}$, respectively. Due to some inaccuracy in the calibration of the EPIC/pn energy scale, a gain correction was also applied (see Appendix A).

As part of the 2014 campaign, Ark 120 was also observed with the High-Energy Transmission Grating (HETG; Canizares et al. 2005) Spectrometer onboard Chandra, on March 17-22. This is the only Chandra observation of Ark 120 to date. Due to scheduling constraints, it was split into three sequences with total exposure of $\sim 120 \mathrm{ks}$, overlapping with the two central XMM-Newton orbits. The data were reprocessed with the CIAO v4.6 software package. Only the first-order spectra were considered for both the Medium- (MEG) and High-Energy Grating (HEG), and the \pm 1 dispersion arms were combined. Only a modest $(\sim 10 \%)$ flux variation was observed between the individual sequences, whose spectra were therefore merged into a single one for each grating. This yielded a total of $10.2 \times 10^{4}$ (MEG; $0.7-5 \mathrm{keV}$ ) and $4.7 \times 10^{4}$ (HEG; $1-8 \mathrm{keV}$ ) counts. The background contribution to the count rate was negligible. The resulting spectra were initially binned to $\Delta \lambda=20$ (MEG) and $10 \mathrm{~m} \AA$ (HEG), which roughly correspond to the full width at half-maximum (FWHM) nominal resolution. To preserve the uniform spacing in wavelength units, in the 
Table 1. Observation log of the X-ray spectra analyzed in this work.

\begin{tabular}{|c|c|c|c|c|c|}
\hline Mission & Obs. ID & Obs. Start (UTC) & Obs. End (UTC) & Exp. $^{a}$ & $\mathcal{C}^{b}\left(\mathrm{~s}^{-1}\right)$ \\
\hline \multicolumn{6}{|c|}{2014 March campaign } \\
\hline XMM-Newton & 0721600201 & 2014 Mar $18-08: 52: 49$ & 2014 Mar $19-21: 26: 23$ & 81,613 & $27.14 \pm 0.02$ \\
\hline$X M M-N e w t o n$ & 0721600301 & 2014 Mar 20 - 08:58:47 & 2014 Mar $21-21: 17: 21$ & 83,875 & $22.65 \pm 0.02$ \\
\hline$X M M-N e w t o n$ & 0721600401 & 2014 Mar $22-08: 25: 17$ & 2014 Mar $23-20: 54: 18$ & 82,388 & $25.23 \pm 0.02$ \\
\hline$X M M-N e w t o n$ & 0721600501 & 2014 Mar $24-08: 17: 19$ & 2014 Mar $25-21: 00: 54$ & 81,902 & $22.78 \pm 0.02$ \\
\hline Chandra & 16539 & 2014 Mar $17-07: 49: 04$ & 2014 Mar 18 - 02:13:52 & 63,016 & $0.402 \pm 0.003$ \\
\hline Chandra & 15636 & 2014 Mar 21 - 04:39:50 & 2014 Mar $21-07: 54: 29$ & 10,239 & $0.342 \pm 0.010$ \\
\hline Chandra & 16540 & 2014 Mar $22-13: 56: 19$ & 2014 Mar $23-04: 01: 17$ & 47,257 & $0.376 \pm 0.004$ \\
\hline \multicolumn{6}{|c|}{ Previous observations } \\
\hline$X M M-N e w t o n$ & 0147190101 & 2003 Aug $24-05: 35: 43$ & 2003 Aug $25-12: 44: 33$ & 78,166 & $26.37 \pm 0.02$ \\
\hline$X M M-N e w t o n$ & 0693781501 & 2013 Feb $18-11: 39: 53$ & 2013 Feb $19-23: 54: 11$ & 87,721 & $10.33 \pm 0.01$ \\
\hline Suzaku & 702014010 & 2007 Apr 01 - 18:07:26 & 2007 Apr $03-21: 43: 25$ & 100,864 & $2.186 \pm 0.003$ \\
\hline
\end{tabular}

Notes. ${ }^{a}$ Net exposure in seconds. ${ }^{b}$ Source count rate over the $0.3-10 \mathrm{keV}$ (XMM-Newton/pn), 1-8 keV (Chandra/HEG), and 0.6-10 keV (Suzaku/XIS 03) bands.

HETG fits we made use of the $C$-statistic (Cash 1979), although the number of counts in each bin exceeds 25 over most of the range covered by the present analysis.

In this study we also considered the two previous XMM-Newton observations of Ark 120, taken respectively on 2003 August 24-25 (Vaughan et al. 2004) and on 2013 February 18-19 (again in combination with NuSTAR; Matt et al. 2014), as well as the Suzaku one, performed on 2007 April 1-3. The 2003 and 2013 XMM-Newton data were reduced with the same criteria adopted above. The Suzaku spectra were re-extracted following the steps described in Nardini et al. (2011). For simplicity, here we used the single, merged spectrum from the two operating front-illuminated detectors (i.e., XIS 0 and XIS 3; Koyama et al. 2007), rebinned by a factor of four to 512 energy channels and further grouped to a minimum of 50 counts per bin. The observation log for all the data sets employed in this work is provided in Table 1. The spectral analysis was performed using the XSPEC v12.9 package. All the fit uncertainties correspond to the $90 \%$ confidence level $\left(\Delta \chi^{2}\right.$ or $\left.\Delta C=2.71\right)$ for the single parameter of interest. Unless otherwise stated, the energy of the emission lines always refers to the rest frame of Ark 120. A concordance cosmology with $H_{0}=70 \mathrm{~km}$ $\mathrm{s}^{-1} \mathrm{Mpc}^{-1}, \Omega_{m}=0.27$, and $\Omega_{\Lambda}=0.73$ (Hinshaw et al. 2013) was assumed throughout.

\section{SPECTRAL ANALYSIS}

\subsection{Chandra/HEG spectrum}

We started our analysis from the Chandra observation, since the high-resolution data would allow us to determine the intrinsic width of any narrow FeK emission component. Here we concentrate on the 3-8 keV band of the HEG spectrum only, referring to Reeves et al. (2016) for a thorough discussion of the soft X-ray properties. We first fitted the continuum with a simple power law, after excluding the observed $5-7 \mathrm{keV}$ energy range. A more complex model is not required, due to the absence of any spectral curvature above $3 \mathrm{keV}$ (Appendix B). This leaves clear residuals in emission at $6.0-7.2 \mathrm{keV}$ in the rest frame, the most prominent of which is a narrow line at $\sim 6.4 \mathrm{keV}$ (Figure 1). A pair of broader and shallower features are seen redwards and bluewards of this core, and a hint of a further line is present at $\sim 7.1 \mathrm{keV}$. Based on the visual inspection, we therefore included four Gaussian profiles, letting the centroid energy, width, and normalization free to vary for each of them. We also adopted the finer resolution of $5 \mathrm{~m} \AA$ (or $15 \mathrm{eV}$ at $6 \mathrm{keV}$ ), which turns out to be more appropriate to fit such a rich and irregular Fe K spectrum. This model gives a statistic of $C / \nu=509 / 504$, and the best-fit line parameters are summarized in Table 2. For ease of illustration, at this stage we dub the three major lines as $\mathrm{K} \alpha_{\text {red }}, \mathrm{K} \alpha_{\text {core }}$, and $\mathrm{K} \alpha_{\text {blue }}$, respectively; their actual nature will be discussed in more detail later on. The faintest line at $\sim 7.1 \mathrm{keV}$ (marginally consistent with Fe K $\beta$ ) is barely significant with its $\sim 25$ counts (against $\sim 115$ for $\mathrm{K} \alpha_{\text {core }}, \sim 80$ for $\mathrm{K} \alpha_{\text {red }}$, and $\sim 65$ for $\mathrm{K} \alpha_{\text {blue }}$ ). Indeed, after its removal, the change in the fit statistic is completely negligible. As the HEG spectrum becomes rather noisy beyond $7 \mathrm{keV}$ (with $<10$ counts per $5-\mathrm{m} \AA \mathrm{bin}$ ), we do not consider this feature any further in this section. Of the three provisional K $\alpha$ components, the red one is definitely the most puzzling, since it does not have the typical aspect of the gravitationally redshifted, extended wing of disk lines (e.g., Miller 2007). On the other hand, its energy of $6.13 \mathrm{keV}$ and equivalent width (EW) 
Table 2. Fe K lines detected in the Chandra/HEG spectrum.

\begin{tabular}{lccccc}
\hline \hline Line & $E(\mathrm{keV})$ & $\sigma(\mathrm{eV})$ & Norm. & $\mathrm{EW}(\mathrm{eV})$ & $\Delta C$ \\
\hline $\mathrm{K} \alpha_{\text {red }}$ & $6.128_{-0.063}^{+0.121}$ & $83_{-50}^{+317}$ & $2.90_{-1.60}^{+4.97}$ & $53_{-31}^{+32}$ & 14.0 \\
$\mathrm{~K} \alpha_{\text {core }}$ & $6.416_{-0.017}^{+0.016}$ & $43_{-15}^{+22}$ & $4.63_{-1.58}^{+1.56}$ & $91_{-25}^{+28}$ & 54.8 \\
$\mathrm{~K} \alpha_{\text {blue }}$ & $6.679_{-0.043}^{+0.044}$ & $64_{-27}^{+47}$ & $2.99_{-1.39}^{+1.63}$ & $64_{-32}^{+32}$ & 16.0 \\
$\mathrm{~K} \beta$ & $7.136_{-0.069}^{+0.077}$ & $<129$ & $1.46_{-1.19}^{+1.48}$ & $<72$ & 4.3 \\
\hline
\end{tabular}

Notes. See the text for the adopted line nomenclature. Normalizations are in units of $10^{-5}$ photons s $\mathrm{cm}^{-2}$, and uncertainties are given at the nominal $90 \%$ level $(\Delta C=2.71)$. The $3-8 \mathrm{keV}$ continuum is modeled with a simple power law with $\Gamma=1.91 \pm 0.07$. Since the lines are somewhat blended, EWs are computed with respect to the continuum only. The change in the statistic after the line removal $(\Delta C$, last column) is obtained without refitting to avoid meaningless solutions.

of $\sim 55 \mathrm{eV}$ also rule out the possibility that this is just the Compton shoulder (Yaqoob \& Murphy 2011, and references therein) of the $\mathrm{K} \alpha$ emission feature from neutral iron at $6.4 \mathrm{keV}$. Such an interpretation, in fact, is straightforward for the least ambiguous $\mathrm{K} \alpha_{\text {core }}(\mathrm{EW} \sim 90 \mathrm{eV})$, whose properties can thus be highly informative. Its width $\sigma_{\text {core }}=43_{-15}^{+22}$ $\mathrm{eV}$ corresponds to a FWHM velocity broadening of $4700_{-1500}^{+2700} \mathrm{~km} \mathrm{~s}^{-1}$, in good agreement with the values obtained for $\mathrm{H} \beta$ in the optical (5800-6100 $\mathrm{km} \mathrm{s}^{-1}$; Wandel et al. 1999; Marziani et al. 2003) and for the soft X-ray lines from He-like species (Reeves et al. 2016). The centroid of $\mathrm{K} \alpha_{\text {blue }}$ at $6.68 \mathrm{keV}$, instead, would be compatible with a Fe xxV $\mathrm{K} \alpha$ identification (but see below). Its slightly larger width compared to $\mathrm{K} \alpha_{\text {core }}$ could be due to the emitting gas being closer to the illuminating source. However, a common velocity broadening of $\sim 5500 \mathrm{~km} \mathrm{~s}^{-1}$, strikingly similar to the $\mathrm{H} \beta$ one, returns an indistinguishable fit $(C / \nu=510 / 505)$.

We can safely exclude that the $\mathrm{K} \alpha$ emission core is unresolved; once we fix the width of $\mathrm{K} \alpha_{\text {core }}$ to zero, the fit quality declines by $\Delta C / \Delta \nu \sim 11 / 1$. This alternative model is not satisfactory on qualitative grounds either, since both $\mathrm{K} \alpha_{\text {red }}$ and $\mathrm{K} \alpha_{\text {blue }}$ are shifted in energy and distorted into a blended, formless profile (compare with Figure 1). The confidence contours derived for $\mathrm{K} \alpha_{\text {core }}$ are shown in Figure 2. In spite of some degeneracy with the adjacent features, the neutral Fe $\mathrm{K} \alpha$ emission line is resolved at a $\sim 3 \sigma$ significance level. Its $\mathrm{EW}$ of $91_{-25}^{+28} \mathrm{eV}$ is broadly consistent with, yet somewhat larger than what expected in the context of the X-ray Baldwin effect (Iwasawa \& Taniguchi 1993). Considering the apparent anti-correlation of the $\mathrm{Fe} \mathrm{K} \alpha$ equivalent width with either the 2-10 keV X-ray luminosity $\left(\sim 10^{44} \mathrm{erg} \mathrm{s}^{-1}\right.$ in Ark 120$)$ or the X-ray to Eddington luminosity ratio $\left(\sim 5 \times 10^{-3}\right)$, we should observe a value up to two times smaller (Bianchi et al. 2007; Shu et al. 2010). In principle, this leaves room for a hidden disk-line component, possibly asymmetric and contributing to some extent to both $\mathrm{K} \alpha_{\text {red }}$ and $\mathrm{K} \alpha_{\text {blue }}$. Indeed, it is worth noting that these structures can be excellently reproduced $(C / \nu=513 / 506)$ as the red and blue horns of a disk line (e.g., Laor 1991) centered at $6.41 \pm 0.05 \mathrm{keV}$, arising at a distance of $\sim 200$ gravitational radii $\left(r_{\mathrm{g}}=G M / c^{2}\right.$, where the black hole mass $M$ is $\sim 1.5 \times 10^{8} M_{\odot}$ in Ark 120; Peterson et al. 2004; Ho \& Kim 2015) and seen at high inclination $\left(i \sim 80^{\circ}\right)$. An almost edge-on configuration would be hard to reconcile with the bare appearance of Ark 120 . The geometry, however, is totally unconstrained $\left(\Delta C=1\right.$ for $r_{\mathrm{in}}=6 r_{\mathrm{g}}$ and $\left.i=30^{\circ}\right)$. The narrow K $\alpha$ core would still be resolved at $\sigma=31_{-12}^{+19} \mathrm{eV}$, fully consistent with the value in Table 2. We then argue that a width of $40 \mathrm{eV}$ is the most suitable for the subsequent analysis of the lower-resolution $X M M-N e w t o n$ and Suzaku spectra.

\subsection{XMM-Newton spectra (2014)}

As done with Chandra, we obtained an initial assessment of iron emission in the four 2014 XMM-Newton spectra by ignoring the observed 5-7 keV band and fitting the remaining 3-10 keV continuum with a power law. The resulting data/model ratios are shown in Figure 3. Even if the overall Fe K profile naturally looks smeared compared to the high-resolution HEG view, the presence of clear residuals on both sides of the main 6.4-keV line is fully confirmed. The most striking difference is that the blue excess seems to extend at least up to $\sim 7 \mathrm{keV}$, where no obvious feature is detected in the Chandra spectrum (Figure 1). This might be ascribed to the much larger EPIC/pn collecting area, which overrides the photon noise, but also short-term intensity fluctuations actually play an important role (see Section 4). Indeed, if we superimpose the HEG best fit on the $X M M-N e w t o n$ data, only allowing for a cross normalization factor (of 0.7-0.8), the former $\mathrm{K} \alpha_{\text {red }}$ and $\mathrm{K} \alpha_{\text {blue }}$ are somewhat overestimated. Conversely, the strength of the core is perfectly matched, again suggesting that the shallower features are affected by noise and/or variability (Figure 4). We first ignored the findings from Chandra and tested a model simply consisting of a power law plus two broad Gaussian profiles (whose widths were tied as $\sigma_{2}=E_{2} \sigma_{1} / E_{1}$ ). On statistical grounds, this fit would be already acceptable, returning a $\chi^{2}$ of $218,264,249$, and 214 for 226 degrees of freedom (d.o.f.) in the four spectra (labeled as 2014a, -b, -c, -d hereafter). The implied FWHM is twice as large as the $\mathrm{H} \beta$ one, and would call for an origin inside the 


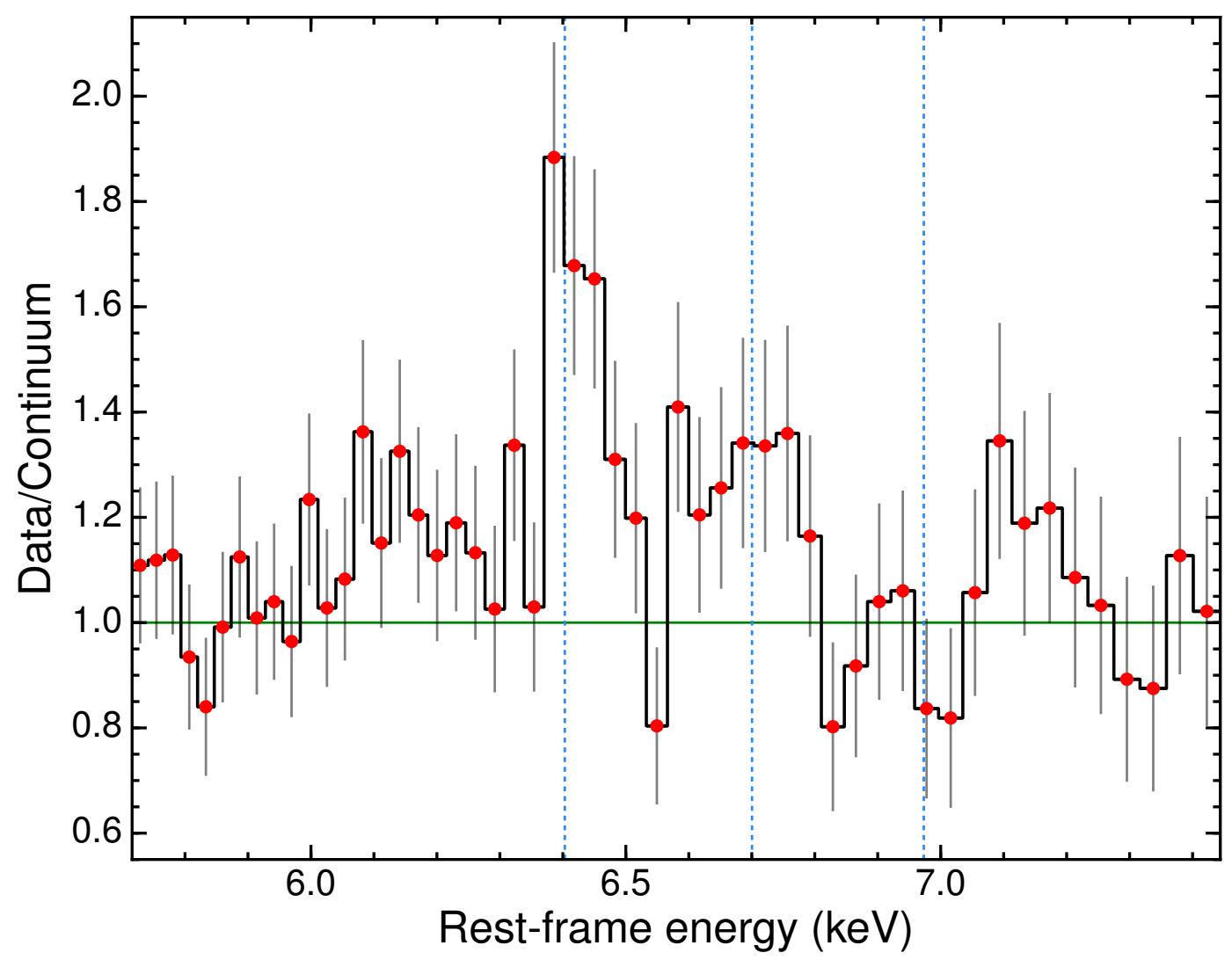

Figure 1. Data to model ratio of the Chandra/HEG spectrum in the Fe K band, obtained by fitting the continuum with the exclusion of the observed 5-7 keV range. A complex emission pattern clearly emerges, dominated by the narrow $(\sigma \simeq 40 \mathrm{eV})$, resolved feature peaking around $6.4 \mathrm{keV}$. The vertical dashed lines correspond to the expected energies of the Fe I, Fe XXV, and Fe XXVI K $\alpha$ transitions. The data are binned to the FWHM resolution.

optical broad-line region (BLR). However, this scenario is at odds with the high-resolution Chandra spectrum, whose availability then proves to be crucial for a sound Fe K decomposition.

We therefore resorted to the HEG-based information on the most likely intrinsic FWHM of the 6.4-keV K $\alpha$ core, forcing the two lines to be substantially narrower $\left(\sigma_{1}=40 \mathrm{eV}\right)$. After re-fitting, the statistic in the single data sets deteriorates by $\Delta \chi^{2}=17,42,32$, and 25 for one extra degree of freedom (d.o.f.), respectively. We then included a third, broad Gaussian line, whose properties $\left(E_{\mathrm{G}} \sim 6.45 \mathrm{keV}\right.$ and $\sigma_{\mathrm{G}} \sim 260 \mathrm{eV}$ on average; Table 3$)$ do not vary significantly across the four spectra. Not only is this fit completely successful, but it is also marginally better than the initial, phenomenological one in each sequence, with $\chi^{2}$ now equal to $215,258,239$, and 211 (for $\nu=224$ ). As the energies and widths of all the lines are always consistent within the errors, we also analyzed the merged spectrum $(2014 m$, obtained by combining the four observations), in order to benefit from its higher quality. The statistical improvement yielded by the addition of a broad Gaussian over the initial, ill-informed model is definitely robust when referred to $2014 m$, with $\Delta \chi^{2} / \Delta \nu=-21 /-2$. Moreover, the position of the minor narrow peak moves from the unusual energy of $6.86-6.91 \mathrm{keV}$ and is now virtually coincident with that of the Fe XXVI K $\alpha$ transition at $6.97 \mathrm{keV}$. The physical explanation for a line with a FWHM of $\sim 30,000 \mathrm{~km} \mathrm{~s}^{-1}$ is yet unclear. Applying the standard virial arguments, this emission feature should arise at a distance of $\sim 100 r_{\mathrm{g}}$, which is the scale of the mid (outer) accretion disk, rather than that of the BLR.

Hence, it is worth considering an alternative model where the residual structures are described through a disk line, also given some previous claims based on the 2007 Suzaku observation (Nardini et al. 2011; Patrick et al. 2011; see also Vaughan et al. 2004 for earlier evidence). We tested several different relativistic line profiles, which all give the same results. Then we only focus on the latest and most sophisticated one, relline (Dauser et al. 2010). With its $\mathrm{EW}<100 \mathrm{eV}$, this feature is not as prominent as in some other, well-studied Seyfert galaxy. In NGC 1365, for instance, the broad $\mathrm{Fe} \mathrm{K} \alpha$ component might reach an $\mathrm{EW}$ of several hundreds $\mathrm{eV}$, and also its overall flux is almost an order of magnitude greater than here (Risaliti et al. 2009). For this reason, not all of the line parameters in 


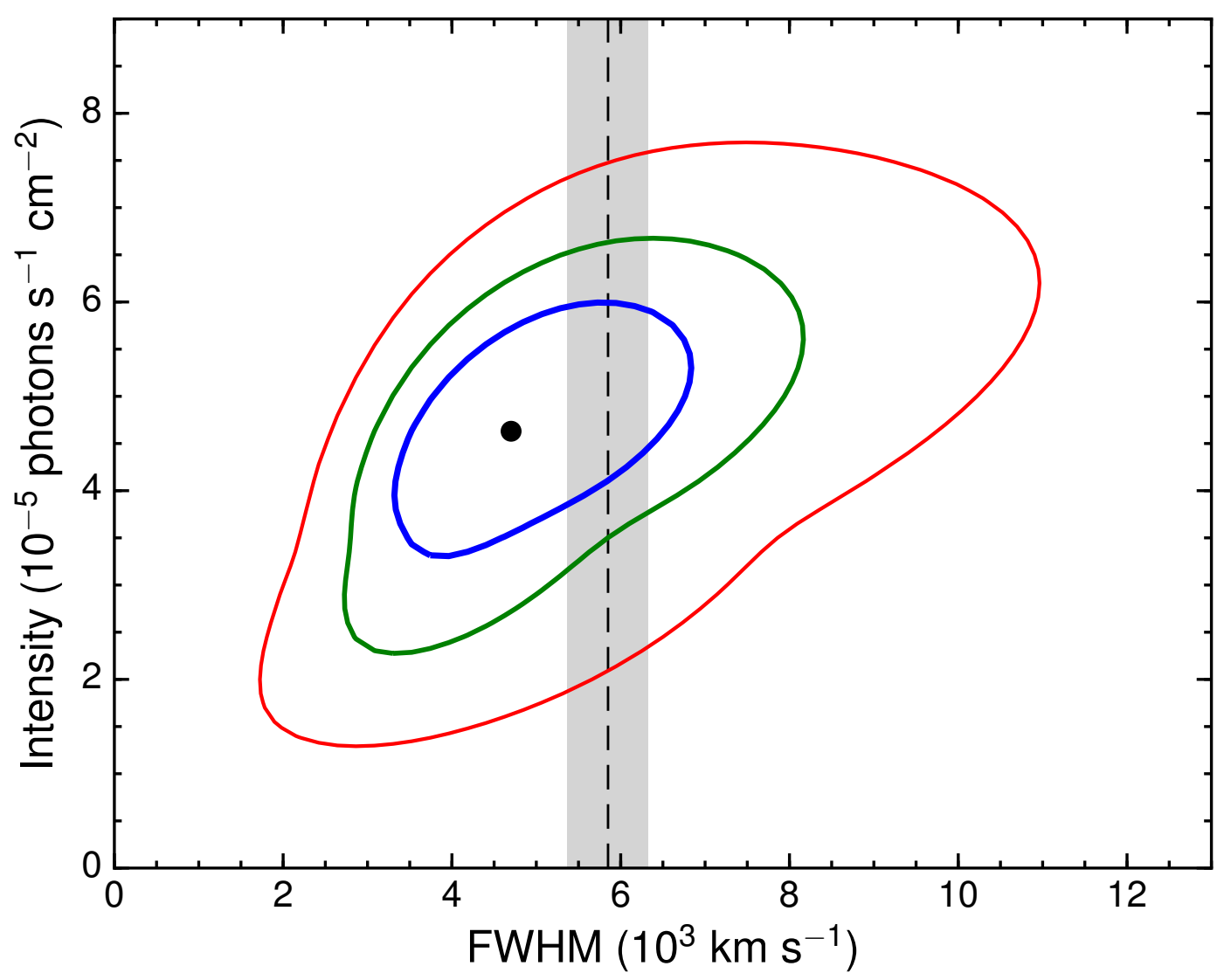

Figure 2. Intensity versus velocity width contours at the nominal 68,90 , and $99 \%$ confidence levels $(\Delta C=2.3,4.61$, and 9.21 , respectively) for the Fe K $\alpha$ emission feature at $\sim 6.4 \mathrm{keV}$. Energy, width, and normalization of the red and blue components on each side of the core were allowed to vary. For comparison, the dashed line and the shaded area indicate the FWHM of the optical $\mathrm{H} \beta$ line $\left(5850 \pm 480 \mathrm{~km} \mathrm{~s}^{-1}\right.$; from Wandel et al. 1999).

Ark 120 can be well constrained simultaneously. We thus assumed no SMBH $\operatorname{spin}^{1}$, a disc inclination of $i=30^{\circ}$, and an $\epsilon \propto r^{-q}$ emissivity law, with $q=3$ (i.e., the Newtonian limit towards infinity). While the inclination conforms with the orientation of the host galaxy $\left(26^{\circ}\right.$; Nordgren et al. 1995), the other two conditions are physically consistent with the best-fit estimates for the disk inner radius, which is of the order of $r_{\mathrm{in}} \sim 60 r_{\mathrm{g}}$ (Table 3). The rest-frame energy of the relline component $\left(E_{\mathrm{R}} \simeq 6.50 \mathrm{keV}\right)$ denotes a mildly ionized reflecting surface. Statistically, the 'disk line' model is impossible to distinguish from the 'broad Gaussian' one, with a difference of $\Delta \chi^{2}=1$ for the same number of d.o.f. (224) in $2014 \mathrm{~m}$. The spectral decomposition of the three lines is shown in Figure 4, where the relline shape is only moderately skewed, since the effects of both gravitational redshift and relativistic beaming are rather weak at the large distances involved.

\subsection{Previous observations}

A broad Fe K emission component is clearly involved in the 2014 XMM-Newton spectra of Ark 120 . In order to gain some more insights into its nature, we need to investigate its behavior over timescales of years. The analysis of the two previous XMM-Newton observations of Ark 120 is very useful in this sense. The spectrum of 2003 August, unfortunately, does not add much information. Indeed, its broadband shape and flux level are nearly identical to those observed in the first orbit of the 2014 campaign down to $\sim 1 \mathrm{keV}$, where the soft excess exhibits a more pronounced roll-over compared to 2014a (Vaughan et al. 2004). Also the emission lines in the Fe K band are in keeping with the best-fit parameters found about 11 years later in each model (see Table 3 and Figure 5, left panel). The broad feature, in both the Gaussian and disk line cases, does not show any significant variation. Much more interesting is the 2013 observation (performed only 13 months before the large campaign), as this is the only one so far that has caught Ark 120 in a markedly different flux and spectral state (Matt et al. 2014). The 3-10 keV continuum is fainter

\footnotetext{
1 The dimensionless spin parameter is defined as $a^{*}=c J / G M^{2}$, where $J$ and $M$ are the black hole's angular momentum and mass.
} 


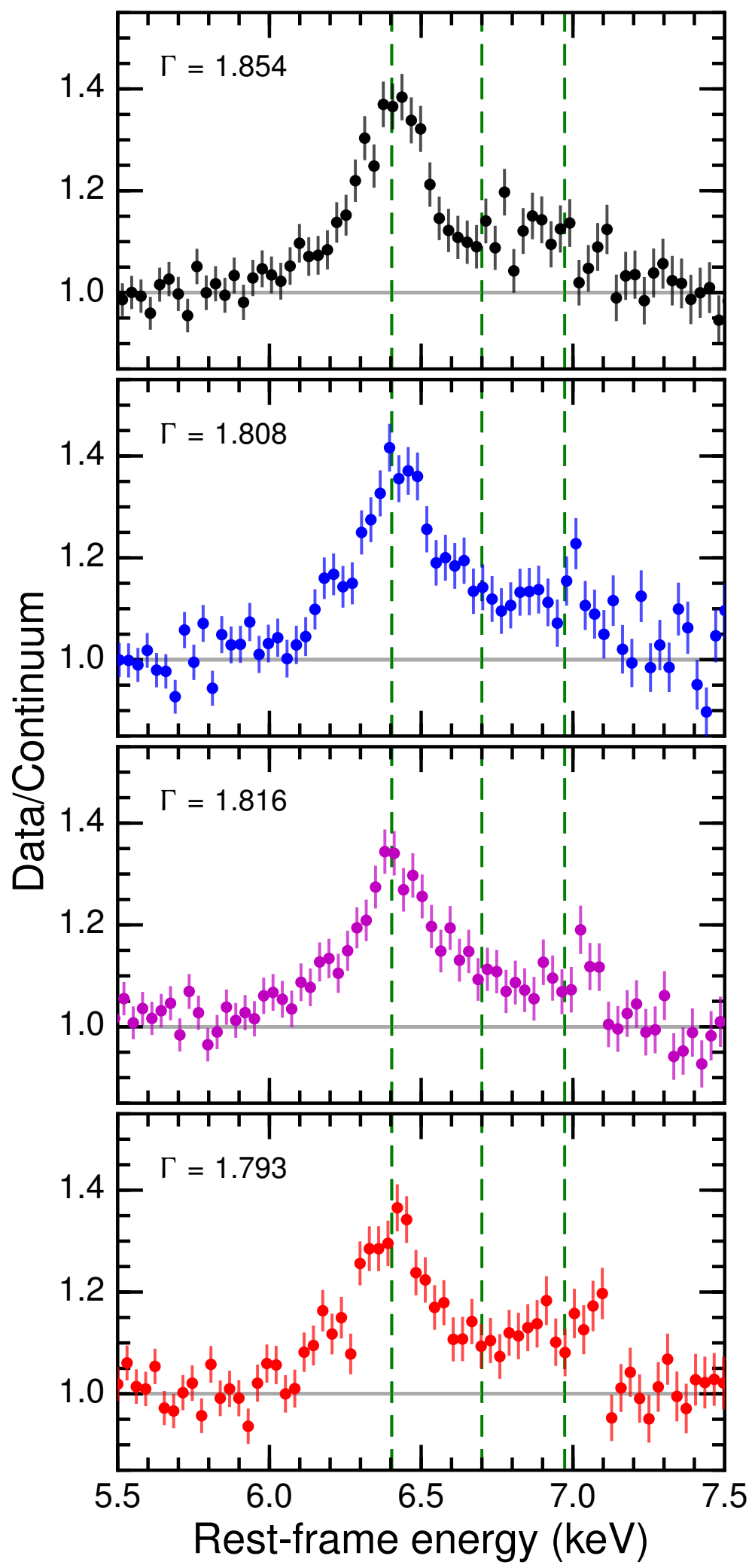

Figure 3. Fe K emission complex in the four consecutive XMM-Newton observations of 2014 March, again obtained as the ratio between the data and the power-law continuum fitted at 3-5 plus $7-10 \mathrm{keV}$ (the best value of the photon index is reported in the left-hand top corner). Compared to the simultaneous, high-resolution Chandra spectrum, the 6.4-keV feature is of course much broader and less prominent, yet the red and blue excesses are still clearly present. Differently from the HEG case, the latter extends with no discontinuity up to at least $7 \mathrm{keV}$, not only due to the much better signal-to-noise of the XMM-Newton spectra, but also to variability (see Section 4). The data are rebinned to a constant $30-\mathrm{eV}$ resolution, and that the energies are gain-corrected. 


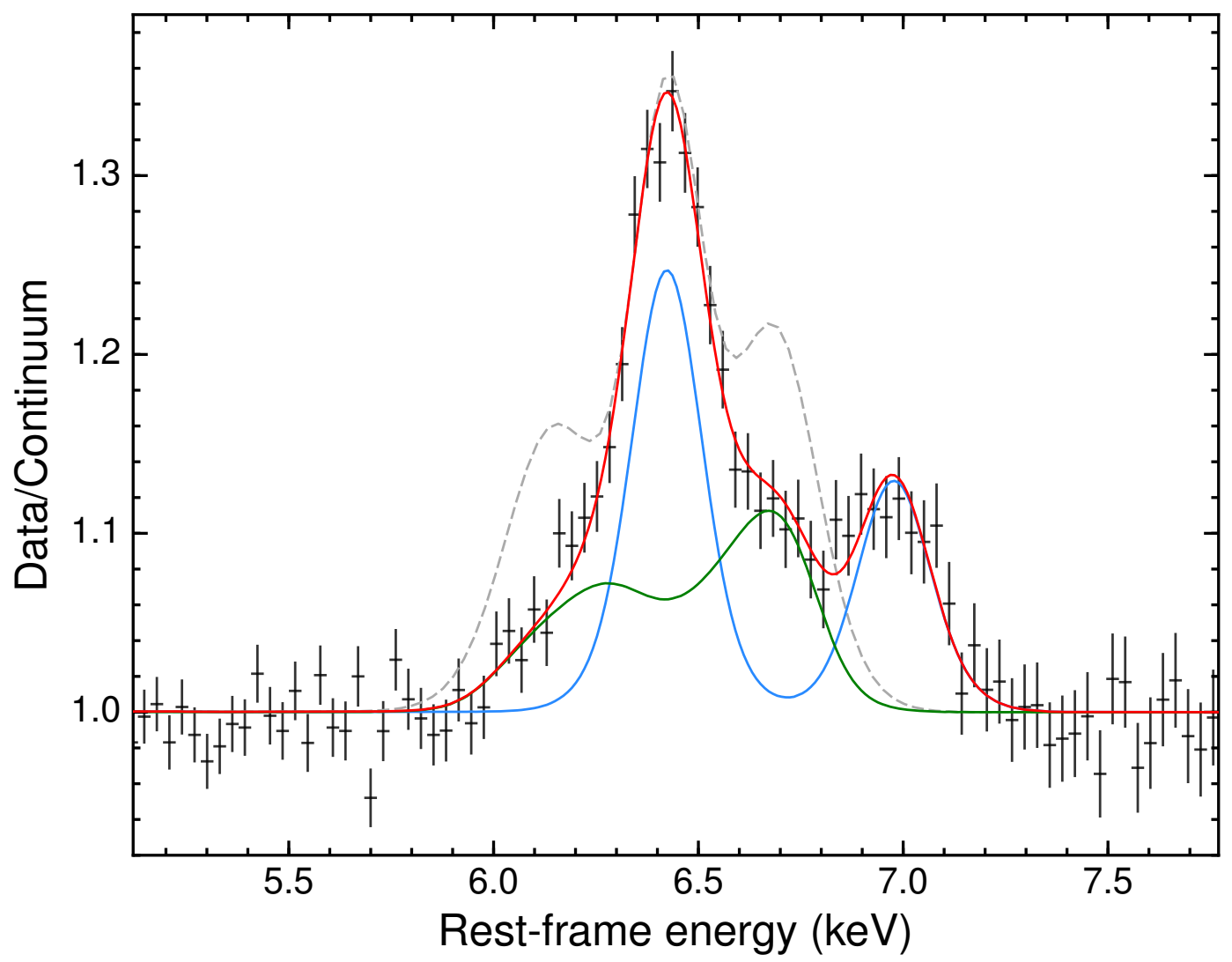

Figure 4. Spectral decomposition of the Fe K emission complex in the 2014m XMM-Newton spectrum (averaged over the entire campaign) when fitted with two narrow Gaussian lines at $E_{1} \simeq 6.41 \mathrm{keV}$ and $E_{2} \simeq 6.97 \mathrm{keV}$ (in turquoise) plus a relativistically distorted line from the mildly ionized accretion disk (in green). The latter feature apparently originates at several tens of gravitational radii from the SMBH, and cannot be statistically preferred to a very broad (FWHM $\sim 30,000 \mathrm{~km} \mathrm{~s}^{-1}$ ) Gaussian. The dashed line shows the overall profile of the three K $\alpha$ components (red, core, and blue) in the Chandra/HEG best fit, which is a first hint of short-term variability.

by a factor of $\sim 1.7-2$ with respect to all the other epochs, flatter $(\Gamma \sim 1.7)$, and vaguely more convex, with no obvious break energy between the soft excess and the hard power law. On the other hand, the relative strength of the former is very close to the average, once the different X-ray slope is taken into account. Following the continuum decrease, the EW of the two narrow lines grows by a similar factor (consistent within the uncertainties; Table 3), suggesting that there is no response on short ( $<1$ day) timescales, comparable to the observation length. The broad/disk line, instead, clearly deviates from the above trend, since its EW is, if anything, smaller than in the 2003 and 2014 high-flux states. There is no compelling evidence for a red wing to the $6.4-\mathrm{keV}$ feature, presumably because it is too faint, nor for any other kind of excess (Figure 5, middle panel). An additional Gaussian is therefore rather narrow $(\sigma \sim 80 \mathrm{eV}$, although the upper limit is poorly constrained), and simply fits some minor residuals at $6.6-6.7 \mathrm{keV}$. Such a drastic width change from 2013 to 2014 is dubious, leaving once more the interpretation in terms of a (variable) fluorescence feature from the accretion disk as the most convincing one.

The relline component in the 2013 spectrum falls at a slightly higher energy $\left(E_{\mathrm{R}} \simeq 6.55 \mathrm{keV}\right)$, and its EW of $\sim 65 \mathrm{eV}$ is comparable to the $2014 \mathrm{~m}$ one. This means that its intensity is roughly subject to the same attenuation experienced by the continuum. Other than this, the main discrepancy involves the disk inner radius, which moves much further out $\left(r_{\mathrm{in}} \gtrsim 100 r_{\mathrm{g}}\right)$. This is mostly due to the lack of a strong red wing, which removes the necessity of any asymmetry in the line profile, so tending to a Newtonian regime. Nevertheless, if both $E_{\mathrm{R}}$ and $r_{\text {in }}$ are fixed to the values of $2014 m$, the fit statistic worsens by only $\Delta \chi^{2} / \Delta \nu=5 / 2$. While the ionization state of the gas, hence the line energy, can reasonably differ from 2013 to 2014, it is not clear which mechanism could be responsible for a recession of the disk inner boundary. In any case, the broad Fe K $\alpha$ component detected in Ark 120 is apparently characterized by sizeable flux variability on timescales of just one year.

The Suzaku observation performed in 2007 April conveniently covers the monitoring gap between the XMM-Newton visits, which are separated by either one or ten years. Moreover, the recorded 3-10 keV power-law flux of $\sim 2.2 \times 10^{-11}$ 
Table 3. Fits to all the XMM-Newton and Suzaku spectra of Ark 120 in the $3-10 \mathrm{keV}$ band.

\begin{tabular}{|c|c|c|c|c|c|c|c|c|}
\hline Obs. & $2014 \mathrm{a}$ & $2014 b$ & $2014 \mathrm{c}$ & $2014 d$ & $2014 m$ & 2003 & 2013 & 2007 \\
\hline \multicolumn{9}{|c|}{ powerlaw +2 zgauss (narrow) + zgauss (broad) } \\
\hline$\Gamma$ & $1.84_{-0.01}^{+0.01}$ & $1.80_{-0.02}^{+0.01}$ & $1.80_{-0.01}^{+0.01}$ & $1.78_{-0.01}^{+0.01}$ & $1.79_{-0.01}^{+0.01}$ & $1.83_{-0.01}^{+0.02}$ & $1.66_{-0.02}^{+0.02}$ & $1.95_{-0.02}^{+0.02}$ \\
\hline$K$ & $1.13_{-0.02}^{+0.03}$ & $0.96_{-0.02}^{+0.03}$ & $1.10_{-0.03}^{+0.02}$ & $0.97_{-0.02}^{+0.03}$ & $1.02_{-0.01}^{+0.01}$ & $1.13_{-0.02}^{+0.03}$ & $0.44_{-0.01}^{+0.02}$ & $1.05_{-0.04}^{+0.04}$ \\
\hline$E_{1}$ & $6.39_{-0.01}^{+0.03}$ & $6.40_{-0.02}^{+0.02}$ & $6.40_{-0.03}^{+0.03}$ & $6.39_{-0.03}^{+0.02}$ & $6.41_{-0.01}^{+0.01}$ & $6.37_{-0.03}^{+0.03}$ & $6.40_{-0.03}^{+0.03}$ & $6.40_{-0.01}^{+0.02}$ \\
\hline $\mathrm{EW}_{1}$ & $65_{-17}^{+17}$ & $56_{-20}^{+21}$ & $39_{-18}^{+21}$ & $52_{-22}^{+22}$ & $47_{-9}^{+10}$ & $42_{-17}^{+18}$ & $99_{-49}^{+57}$ & $60_{-14}^{+16}$ \\
\hline$E_{2}$ & $6.90_{-0.08}^{+0.08}$ & $6.98_{-0.05}^{+0.06}$ & $7.00_{-0.06}^{+0.05}$ & $6.97_{-0.05}^{+0.04}$ & $6.98_{-0.03}^{+0.03}$ & $7.00_{-0.04}^{+0.04}$ & $7.00_{-0.04}^{+0.03}$ & $6.96_{-0.03}^{+0.04}$ \\
\hline $\mathrm{EW}_{2}$ & $20_{-16}^{+18}$ & $25_{-16}^{+17}$ & $20_{-12}^{+12}$ & $30_{-14}^{+17}$ & $23_{-6}^{+7}$ & $27_{-12}^{+11}$ & $46_{-11}^{+12}$ & $34_{-15}^{+16}$ \\
\hline$E_{\mathrm{G}}$ & $6.46_{-0.16}^{+0.15}$ & $6.48_{-0.05}^{+0.11}$ & $6.42_{-0.06}^{+0.07}$ & $6.47_{-0.09}^{+0.13}$ & $6.47_{-0.04}^{+0.05}$ & $6.51_{-0.06}^{+0.09}$ & $6.61_{-0.18}^{+0.01}$ & $6.36_{-0.09}^{+0.08}$ \\
\hline$\sigma_{\mathrm{G}}$ & $290_{-157}^{+134}$ & $263_{-86}^{+109}$ & $251_{-70}^{+93}$ & $245_{-87}^{+105}$ & $257_{-45}^{+50}$ & $210_{-68}^{+73}$ & $<229$ & $408_{-90}^{+103}$ \\
\hline $\mathrm{EW}_{\mathrm{G}}$ & $68_{-27}^{+28}$ & $95_{-25}^{+24}$ & $86_{-21}^{+22}$ & $71_{-23}^{+24}$ & $86_{-11}^{+12}$ & $83_{-22}^{+23}$ & $<88$ & $164_{-40}^{+43}$ \\
\hline$\chi^{2} / \nu$ & $215 / 224$ & $258 / 224$ & $239 / 224$ & $211 / 224$ & $260 / 224$ & $251 / 224$ & $271 / 224$ & $208 / 219$ \\
\hline \multicolumn{9}{|c|}{ powerlaw +2 zgauss (narrow) + relline } \\
\hline$\Gamma$ & $1.84_{-0.02}^{+0.01}$ & $1.80_{-0.02}^{+0.01}$ & $1.80_{-0.01}^{+0.01}$ & $1.78_{-0.01}^{+0.01}$ & $1.79_{-0.01}^{+0.02}$ & $1.83_{-0.01}^{+0.01}$ & $1.66_{-0.02}^{+0.02}$ & $1.94_{-0.02}^{+0.03}$ \\
\hline$K$ & $1.13_{-0.02}^{+0.03}$ & $0.96_{-0.02}^{+0.02}$ & $1.10_{-0.03}^{+0.02}$ & $0.97_{-0.02}^{+0.02}$ & $1.02_{-0.01}^{+0.01}$ & $1.13_{-0.02}^{+0.03}$ & $0.44_{-0.01}^{+0.02}$ & $1.04_{-0.03}^{+0.04}$ \\
\hline$E_{1}$ & $6.39_{-0.01}^{+0.02}$ & $6.41_{-0.02}^{+0.01}$ & $6.40_{-0.02}^{+0.01}$ & $6.39_{-0.01}^{+0.02}$ & $6.41_{-0.01}^{+0.01}$ & $6.40_{-0.02}^{+0.02}$ & $6.41_{-0.02}^{+0.03}$ & $6.39_{-0.01}^{+0.02}$ \\
\hline $\mathrm{EW}_{1}$ & $71_{-10}^{+11}$ & $70_{-12}^{+13}$ & $52_{-11}^{+13}$ & $62_{-12}^{+12}$ & $58_{-5}^{+5}$ & $48_{-12}^{+12}$ & $79_{-23}^{+28}$ & $70_{-11}^{+11}$ \\
\hline$E_{2}$ & $7.07_{-0.37 p}^{+0.13 p}$ & $6.97_{-0.04}^{+0.05}$ & $6.98_{-0.05}^{+0.05}$ & $6.97_{-0.27 p}^{+0.23 p}$ & $6.97_{-0.03}^{+0.02}$ & $6.99_{-0.03}^{+0.03}$ & $7.00_{-0.04}^{+0.03}$ & $6.96_{-0.03}^{+0.03}$ \\
\hline $\mathrm{EW}_{2}$ & $<19$ & $33_{-10}^{+9}$ & $24_{-8}^{+8}$ & $33_{-11}^{+11}$ & $29_{-4}^{+4}$ & $33_{-10}^{+10}$ & $48_{-11}^{+11}$ & $50_{-10}^{+10}$ \\
\hline$E_{\mathrm{R}}$ & $6.65_{-0.25}^{+0.09}$ & $6.51_{-0.06}^{+0.07}$ & $6.47_{-0.05}^{+0.05}$ & $6.54_{-0.10}^{+0.25}$ & $6.50_{-0.03}^{+0.04}$ & $6.52_{-0.03}^{+0.05}$ & $6.55_{-0.05}^{+0.07}$ & $6.44_{-0.04}^{+0.05}$ \\
\hline$r_{\text {in }}$ & $37_{-13}^{+55}$ & $50_{-19}^{+68}$ & $58_{-21}^{+33}$ & $60_{-33}^{+47}$ & $62_{-12}^{+16}$ & $93_{-32}^{+60}$ & $228_{-130}^{+72 p}$ & $24_{-13}^{+9}$ \\
\hline $\mathrm{EW}_{\mathrm{R}}$ & $83_{-33}^{+34}$ & $78_{-20}^{+25}$ & $71_{-17}^{+20}$ & $61_{-19}^{+26}$ & $69_{-9}^{+9}$ & $72_{-15}^{+18}$ & $63_{-23}^{+30}$ & $142_{-32}^{+32}$ \\
\hline$\chi^{2} / \nu$ & $213 / 224$ & $259 / 224$ & $238 / 224$ & $209 / 224$ & $261 / 224$ & $249 / 224$ & $270 / 224$ & $206 / 219$ \\
\hline
\end{tabular}

Notes. The power-law normalization $(K)$ is given in units of $10^{-2}$ photons $\mathrm{keV}^{-1} \mathrm{~s}^{-1} \mathrm{~cm}^{-2}$ at $1 \mathrm{keV}$. Line energies are in keV, while widths $(\sigma$ and $\mathrm{EW})$ are in $\mathrm{eV}$. The inner radius of the disk $\left(r_{\text {in }}\right)$ is in gravitational radii. The ' $p$ ' flag means that the uncertainty on a given parameter reaches the upper/lower limit allowed by the model. Black hole spin $a^{*}=0$, disk inclination $i=30^{\circ}$, and emissivity index $q=3$ are assumed for the relline component.

$\mathrm{erg} \mathrm{s}^{-1} \mathrm{~cm}^{-2}$ is almost exactly intermediate between the highest (2014c) and the lowest (2013) states, whereas the photon index is steeper than usual $(\Gamma=1.95)^{2}$. We thus applied the same emission-line models employed in the $X M M-N e w t o n$ analysis to the $3-10 \mathrm{keV}$ Suzaku spectrum. The first outcome is that the red excess has a much more appreciable wing-like form, extending down to at least $5.7 \mathrm{keV}$ (Figure 5, right panel). This, in turn, implies that to reproduce this structure with a Gaussian profile (centered at lower energy, $E_{\mathrm{G}}=6.36_{-0.09}^{+0.08} \mathrm{keV}$ ) a width of $\sigma_{\mathrm{G}} \simeq 410$ $\mathrm{eV}\left(\mathrm{FWHM} \sim 45,000 \mathrm{~km} \mathrm{~s}^{-1}\right.$ ) is required, which is probably unphysical unless associated to a face-on disk. Despite the good fit quality $\left(\chi^{2} / \nu=208 / 219\right)$, this is not a viable alternative to the 'disk line' model. The resort to a relline component $\left(a^{*}=0, i=30^{\circ}, q=3\right)$ yet brings a further improvement $\left(\Delta \chi^{2}=-2\right.$ for the same d.o.f.). As expected from the noticeable footprint of gravitational redshift, the disk inner radius moves closer to the innermost stable circular orbit, being about a factor of two smaller than in the $2014 X M M-$ Newton observation $\left(r_{\text {in }} \sim 25\right.$ versus $\left.60 r_{\mathrm{g}}\right)$. Most notably, while the EW of two narrow lines at 6.39 and $6.96 \mathrm{keV}$ modestly grows following the established anti-correlation with the underlying flux, the EW of the disk line remarkably increases to $\sim 140( \pm 30) \mathrm{eV}$. This is twice as large as seen in 2014, against a 20-25\% diminution of the continuum. All the lines' parameters are listed in the last column of Table 3, and are found to be in agreement with the results of Nardini et al. (2011), where a laor kernel (Laor 1991) with $i=40^{\circ}$ was used instead. The Suzaku spectrum then delivers a strong confirmation of a disk line, whose behavior is largely unpredictable when compared to the overall hard X-ray intensity.

The main results of the analysis of the best-quality, time-averaged spectra of Ark 120 can be therefore summarized as follows:

\footnotetext{
2 Differently from the change in the photon index from 2013 to 2014, this might not be entirely physical, given the cross-calibration systematics between the XMM-Newton/EPIC and Suzaku/XIS instruments (e.g., Tsujimoto et al. 2011).
} 


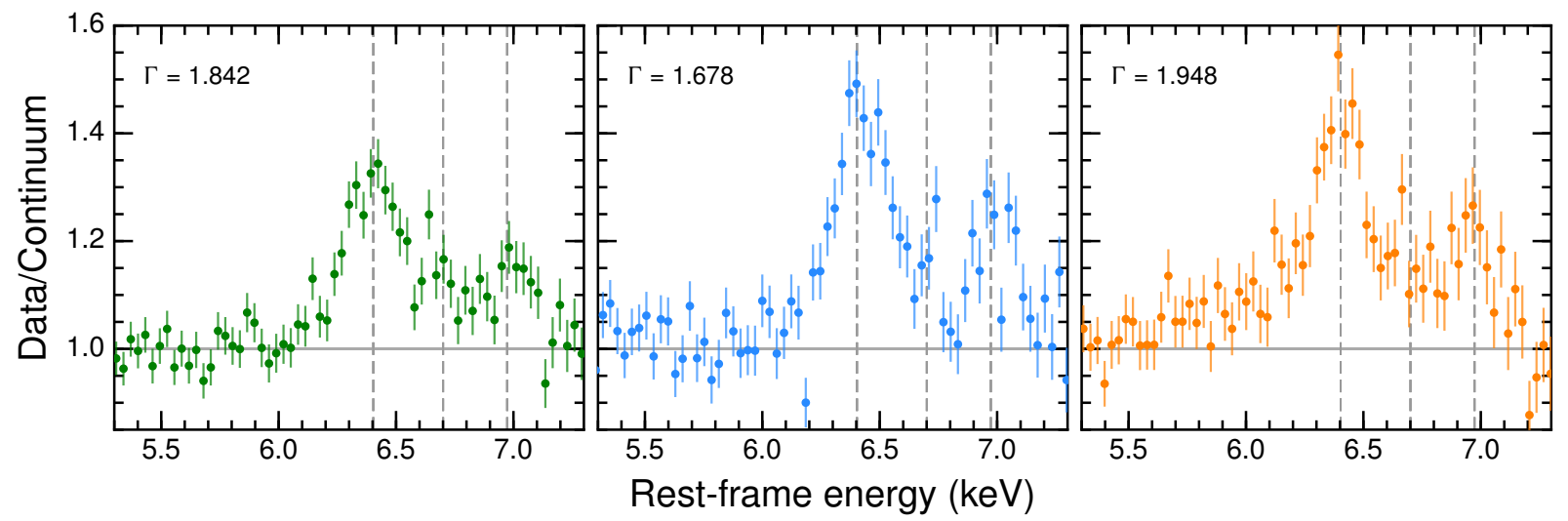

Figure 5. Same as Figure 3 for the 2003, 2013 XMM-Newton, and 2007 Suzaku observations (from left to right). The red Fe K wing is clearly variable in strength and extent on timescales of several years. Also the excess emission between the two narrow features is found at its lowest intensity alongside the wing, supporting their interpretation as signatures of disk reflection.

- The Fe K emission-line complex in Ark 120 displays a rather composite structure, dominated in prominence by the narrow $\mathrm{K} \alpha$ feature from neutral iron peaking at $6.4 \mathrm{keV}$, which is resolved in the Chandra/HEG spectrum. The width value of $\sigma=43_{-15}^{+22} \mathrm{eV}\left(\mathrm{FWHM} \sim 4700 \mathrm{~km} \mathrm{~s}^{-1}\right)$ is commensurate with the velocity broadening of $\mathrm{H} \beta$ in the optical. On both sides of the main core, residual emission is detected in every epoch. In particular, the red, wing-like excess is not compatible with a K $\alpha$ Compton shoulder in terms of energy and equivalent width, thus calling for the presence of a broad and shallow independent feature.

- At the CCD-resolution, yet much higher signal-to-noise of the XMM-Newton and Suzaku spectra, a second narrow line is regularly identified around $7 \mathrm{keV}$, which would be temptingly attributed to Fe XxvI. Indeed, the equivalent width of both the 6.4- and 7-keV features fairly anti-correlates with the continuum flux over a decade (2003 to 2014), implying that their absolute strength is approximately constant, or subject to modest variations at most. Hence these lines appear to be sensitive to the average continuum over long (months to years) timescales only, in keeping with a possible broad-line region origin as suggested by their width.

- The additional residuals, which include the red wing down to $5.7-6 \mathrm{keV}$ as well as a sort of plateau between the two narrow lines, account for up to $\sim 50 \%$ of the total Fe K emission. These components can be jointly fitted through a broad profile, either symmetric $(\sigma \sim 200-300 \mathrm{eV})$ or slightly skewed, as for a disk line emanating from a mild gravitational regime. The latter description is preferred on physical grounds, given the evidence of significant changes in both flux and shape over a period of just one year, which place this feature within several tens to a few hundreds of gravitational radii from the central black hole.

The disk line, even if inconclusive for a precise measurement of the SMBH spin, has a valuable diagnostic potential in the study of the central regions of Ark 120. In the next section we build on the results above and explore the source of the primary X-ray emission through the disk reflection signatures.

\subsection{Reflection geometry and the X-ray source}

The broad Fe K feature in Ark 120 is apparently emitted from a region where relativistic effects are mild. A disk truncation would be anomalous in a source with such an X-ray brightness. Indeed, by taking a disk extended down to the innermost stable circular orbit of a SMBH with maximal spin $\left(a^{*}=0.998, r_{\text {in }}=1.235 r_{\mathrm{g}}\right)$, the fit of the $2014 \mathrm{~m}$ spectrum gets worse but not so dramatically, provided that the exponent of the emissivity law is allowed to adjust to $q=1.4_{-0.4}^{+0.3}$. A flatter emissivity would suggest that the X-ray source is either diffuse or, in the lamp-post approximation, located fairly high above the disk, so that the light bending is not so extreme as to focus most of the illuminating radiation towards the very inner regions (Miniutti \& Fabian 2004; Dauser et al. 2013). In general, reflection off the surface of the accretion disk can be a powerful clue to the structure and properties of the irradiating source. With reflection are designated all the effects of the interaction between X-rays and cold, optically-thick matter (e.g., George \& Fabian 1991; Ross \& Fabian 1993), of which fluorescence from iron and the most abundant lighter elements is a key ingredient. In particular, the combination of photoelectric absorption and Compton backscattering imparts considerable curvature to the incident continuum. To take this into account, we replaced the power law and the disk line in our previous models with a relxill spectrum (v0.4a; García et al. 2014), which encompasses both 
the direct and the reflected components in a self-consistent way. We initially maintained the same set of assumptions adopted for relline (i.e., $a^{*}=0, i=30^{\circ}$, and $q=3$ ), and restricted again the analysis to the $3-10 \mathrm{keV}$ energy range. This allowed us to avail ourselves of a decent continuum window, and yet avoid any heavy contamination from the soft/hard excess. All the seven spectra (six from XMM-Newton plus one from Suzaku) were fitted together tying the energies of the two narrow features, as this is fully justified by the data (whereby the most general case has a probability of chance improvement of 0.48 according to an $F$-test). The outcome is very $\operatorname{good}\left(\chi^{2} / \nu=1632 / 1575\right)$, and provides some interesting indications. The inner radius of the disk is exactly the same as before $\left(r_{\mathrm{in}} \sim 25 r_{\mathrm{g}}\right.$; Table 4) for the Suzaku observation, when the broad Fe K line was stronger. In all the other cases it tends to be higher than the values of Table 3 by a factor of $\sim 2$, but still consistent due to the larger uncertainties. In absolute terms, then, $r_{\text {in }}$ is always at great distance from the innermost stable orbit, irrespective of the SMBH spin.

The two other basic variables of the new model are the ionization parameter (defined as $\xi=L / n r^{2}$, where $L$ is the ionizing luminosity, $n$ the density of the gas, and $r$ its distance from the source), which mainly determines the position of the line, and the reflection fraction $\mathcal{R}$, measured as the ratio between the amount of radiation impinging on the disk and that directly escaping towards infinity (e.g., Dauser et al. 2016). For both quantities the confidence intervals largely overlap across the different epochs, with $\log \left(\xi / \mathrm{erg} \mathrm{cm} \mathrm{s}^{-1}\right)$ ranging from $2.30_{-0.24}^{+0.30}$ (2007) to 2.72 ${ }_{-0.42}^{+0.18}(2014 \mathrm{~b})$, and $\mathcal{R}$ ranging from $0.20_{-0.06}^{+0.12}(2014 \mathrm{~d})$ to $0.33_{-0.09}^{+0.06}(2014 \mathrm{c})$, except for the Suzaku spectrum where $\mathcal{R}=0.56_{-0.16}^{+0.14}$ (Table 4). No correlation is then found between the hard X-ray flux and the ionization state, for which the most common species of iron are Fe XIX-XXV (Kallman et al. 2004). While an exhaustive discussion of the broadband X-ray emission of Ark 120, including the NuSTAR data, is deferred to a forthcoming companion paper (Porquet et al. 2016), we anticipate here that the estimated reflection fraction is far too low to reproduce the observed soft excess. Reflection could still heavily contribute towards the flux rise below $2 \mathrm{keV}$, but this would require distinct properties from those inferred from Fe K fluorescence. Indeed, some caution should be exercised when fitting the whole X-ray spectrum with a reflection model (e.g., Nardini et al. 2012; Walton et al. 2013), since the statistical weight of the broad iron line becomes negligible and the relativistic blurring can be driven to an extreme degree by the smoothness of the continuum ${ }^{3}$. This could hinder our ability to distinguish between reflection components arising from different zones of the disk.

Both the disk emissivity pattern and the reflection fraction depend on the geometry, size, and location of the X-ray corona (Wilkins \& Fabian 2012; Dauser et al. 2013). We have so far assumed an emissivity index $q=3$, which is the canonical value at large distance from an isotropic, pointlike source. As the size of the X-ray emitting region in AGNs is apparently of a few gravitational radii (Mosquera et al. 2013, and references therein), and $r_{\text {in }} \gg r_{\mathrm{g}}$ in our fits, there are no self-consistency issues. However, we can reverse the approach, choosing the source configuration with its incorporated $\epsilon(r)$ and $\mathcal{R}$ first. The simplest and most widely explored approximation is the lamp-post geometry (Matt et al. 1991), for which we used the most recent relxilllp calculations. ${ }^{4}$ The height of the source $(h)$ along the black hole's rotation axis is the new free parameter of the model, which controls the illumination of the accretion disk, hence its emissivity and reflection fraction (through the fixReflFrac = 1 option). Also the lamp-post scenario turns out to be statistically successful, with $\chi^{2} / \nu=1638 / 1575$. While the best-fitting $\xi$ values change by $\sim 10-20 \%$ at most, $h$ and $r_{\text {in }}$ are generally comparable and poorly constrained at several tens of $r_{\mathrm{g}}$ (Table 4). Such a height above the disk, although not affected by the computational shortcomings of relxilllp recently noted by Niedźwiecki et al. (2016), is much more difficult to explain than the nominal inner radius. The latter, in fact, does not entail the truncation of the accretion flow well before the last stable orbit. If the corona is radially extended over the inner disk, the reflection component can be completely smeared through Comptonization even for ordinary temperatures and optical depths (Wilkins \& Gallo 2015). Only reflection from the outer regions would be therefore discernible from the primary continuum. Conversely, X-ray reverberation studies of AGNs suggest that the source lies at only a few $r_{\mathrm{g}}$ from the disk (Emmanoulopoulos et al. 2014). Fixing $r_{\text {in }}$ to $6 r_{\mathrm{g}}$ results in a $\Delta \chi^{2} / \Delta \nu=7 / 7$, but pushes the source further up (with a lower limit of $h>125-230 r_{\mathrm{g}}$ in the $X M M-N e w t o n$ spectra; Table 4 ), while fixing $h$ to $10 r_{\mathrm{g}}$ has appreciable drawbacks $\left(\Delta \chi^{2} / \Delta \nu=59 / 7\right.$; chance probability of $\left.1.2 \times 10^{-9}\right)$. Based on the observed disk line, a lamp-post, on-axis configuration for the X-ray corona then appears unlikely for the unusual height. A careful examination of the subtle Fe K spectral variations over different timescales (from several years down to less than a day) is therefore needed to pinpoint the location of the reflecting gas and of its source of illumination, which is not necessarily one and the same

\footnotetext{
3 This can also be deduced from the comparison between the relxill- and the relline-based (Section 4.1) models at 5-7 keV, the former being worse by $\Delta \chi^{2}=20$ for the same degrees of freedom: $\chi^{2} / \nu=435 / 427$ against $415 / 427$ without refitting. Even the tiniest curvature of the continuum (which is indeed better fitted by relxill at 3-10 keV; see also Appendix B) is followed at the expense of the line profile.

${ }^{4}$ http://www.sternwarte.uni-erlangen.de/ dauser/research/relxill/index.html
} 
Table 4. Key parameters of the disk reflection models.

\begin{tabular}{lccccccc}
\hline \hline Obs. & 2003 & 2007 & 2013 & $2014 \mathrm{a}$ & $2014 \mathrm{~b}$ & $2014 \mathrm{c}$ & $2014 \mathrm{~d}$ \\
\hline \multicolumn{7}{c}{ relxill } & (general) \\
$\Gamma$ & $1.85_{-0.02}^{+0.02}$ & $1.99_{-0.03}^{+0.04}$ & $1.67_{-0.02}^{+0.02}$ & $1.85_{-0.02}^{+0.02}$ & $1.80_{-0.02}^{+0.02}$ & $1.82_{-0.02}^{+0.02}$ & $1.79_{-0.02}^{+0.02}$ \\
$r_{\text {in }}$ & $219_{-119}^{+81 p}$ & $24_{-12}^{+12}$ & $300_{-97}^{+p}$ & $74_{-35}^{+114}$ & $136_{-77}^{+164 p}$ & $103_{-51}^{+197 p}$ & $92_{-45}^{+147}$ \\
$\log \xi$ & $2.33_{-0.14}^{+0.37}$ & $2.30_{-0.24}^{+0.30}$ & $2.70_{-0.31}^{+0.05}$ & $2.51_{-0.32}^{+0.25}$ & $2.72_{-0.42}^{+0.18}$ & $2.30_{-0.19}^{+0.09}$ & $2.45_{-0.35}^{+0.33}$ \\
$\mathcal{R}$ & $0.32_{-0.11}^{+0.11}$ & $0.56_{-0.16}^{+0.14}$ & $0.25_{-0.08}^{+0.07}$ & $0.21_{-0.05}^{+0.13}$ & $0.25_{-0.07}^{+0.06}$ & $0.33_{-0.09}^{+0.06}$ & $0.20_{-0.06}^{+0.12}$ \\
\hline \multicolumn{7}{c}{ relxill1p } & $($ lamp-post) \\
$\Gamma$ & $1.84_{-0.02}^{+0.02}$ & $1.99_{-0.03}^{+0.03}$ & $1.66_{-0.02}^{+0.02}$ & $1.85_{-0.02}^{+0.02}$ & $1.80_{-0.02}^{+0.02}$ & $1.82_{-0.02}^{+0.02}$ & $1.78_{-0.01}^{+0.03}$ \\
$h$ & $164_{-112}^{+136}$ & $25_{-14}^{+32}$ & $202_{-135}^{+98 p}$ & $36_{-22}^{+264 p}$ & $109_{-80}^{+191 p}$ & $118_{-82}^{+132}$ & $43_{-28}^{+257 p}$ \\
$r_{\text {in }}$ & $110_{-104 p}^{+45}$ & $7_{-1 p}^{+18}$ & $145_{-139 p}^{+64}$ & $69_{-63 p}^{+73}$ & $110_{-104 p}^{+52}$ & $68_{-62 p}^{+42}$ & $86_{-80 p}^{+91}$ \\
$\log \xi$ & $2.43_{-0.16}^{+0.28}$ & $2.35_{-0.20}^{+0.36}$ & $2.69_{-0.30}^{+0.08}$ & $2.54_{-0.35}^{+0.22}$ & $2.71_{-0.41}^{+0.18}$ & $2.30_{-0.19}^{+0.10}$ & $2.48_{-0.37}^{+0.30}$ \\
\hline$h_{\text {isco }}$ & $256_{-101}^{+44 p}$ & $26_{-14}^{+32}$ & $300_{-70}^{+p}$ & $239_{-90}^{+61 p}$ & $272_{-73}^{+28 p}$ & $175_{-47}^{+91}$ & $300_{-119}^{+p}$ \\
\hline
\end{tabular}

Notes. While $q=3$ in the general case, in the lamp-post configuration the disk emissivity profile and the reflection fraction $(\mathcal{R})$ are self-consistently determined by the height of the source ( $h$, in gravitational radii). The ionization parameter $(\xi)$ is in units of erg $\mathrm{cm} \mathrm{s}^{-1}$. In both models $a^{*}=0$ and $i=30^{\circ}$ are assumed. Also reported is the height value for a disk extending down to the innermost stable circular orbit $\left(h_{\text {isco }}\right)$.

with the source of the hard X-ray continuum.

\section{FeK EMISSION VARIABILITY}

\subsection{Long timescales (months to years)}

To obtain a more quantitative evaluation of the variability of the intensity of all the Fe $\mathrm{K}$ lines across the various epochs, we reverted back to the relline model and fitted the seven spectra at $3-10 \mathrm{keV}$ with the usual assumptions on spin, inclination, and emissivity index for the disk line $\left(\chi^{2} / \nu=1650 / 1575\right.$; see footnote 3$)$. The results are plotted in Figure 6, and are largely model-independent, since a similar trend would be found by fitting the broad component as a Gaussian $\left(\Delta \chi^{2} \simeq 11\right)$. The strength of the supposed BLR lines at $E_{1}=6.399 \pm 0.006 \mathrm{keV}$ and $E_{2}=6.979 \pm 0.014$ presents little in the way of fluctuations. The latter is consistent with being constant, while the former could be somewhat more variable, although this might be due to the superposition with the disk line, whose exact profile is unknown and hard to disentangle. The relativistic feature, in fact, is definitely erratic. In the 2013 low-flux state, the disk line drops by a factor of two compared to the closest observations, with a significance of $3.5 \sigma$ with respect to the average $2014 m$ spectrum (and $2-3 \sigma$ over the individual sequences). The difference is even more pronounced (a factor of three at $5 \sigma$ ) considering the Suzaku spectrum, which, however, was taken six years earlier.

Since the disk line seems to vary not only in flux but also in shape, and specifically in the extent of the red wing, we further investigated its evolution with time by computing the difference spectrum between the 2014/high and 2013/low states. In order to maximise the signal-to-noise, given that the calibration of the relative energy scales might still be not perfectly matched after the gain correction (Appendix A), we used the merged $2014 \mathrm{~m}$ spectrum. Once the effects of the different hard X-ray continuum slopes are factored out, the outcome is the one illustrated in Figure 7 . The Fe K emission complex clearly underwent a substantial change from 2013 to 2014 . The variable component has an uneven shape, vaguely reminiscent of a double peak, where a rising wing at $6 \mathrm{keV}$ can be easily recognized. Notably, no discrepancy is revealed at the FeXXVI K $\alpha$ energy, while the larger excess is visible around $6.4 \mathrm{keV}$. We can then speculate that part of the neutral-iron fluorescence does not occur at BLR scales, but closer in (possibly on the outskirts of the disk), thus responding to the continuum on shorter timescales. Even in this model-independent perspective, however, the broad emission line unveiled in the spectral analysis undoubtedly plays a major role in the overall Fe $\mathrm{K}$ variability. For comparison, in Figure 7 we also show the profile of a disk line of intensity $2 \times 10^{-5}$ photons s $\mathrm{cm}^{-2}$, centered at $6.6 \mathrm{keV}$, and arising from an annulus between 60 and $120 r_{\mathrm{g}}$ (see Table 3 and Figure 6). Aside from around 6.4-6.5 keV, the agreement with the variable component is excellent.

In this light, it is plausible that different regions of the disk contribute to the Fe $\mathrm{K}$ emission at different times. This would explain the disparity between the values of the disk inner radius returned by the single spectra, and the moderate shifts of the line's energy could be ascribed to the local ionization state of the gas. Once the eventualities of a disk truncation and/or of a high-elevation source are regarded as unlikely (Section 3.4), the broad Fe K line properties imply that, for any reason (e.g., full iron ionization, extreme velocity blurring, Comptonization), the inner disk does 


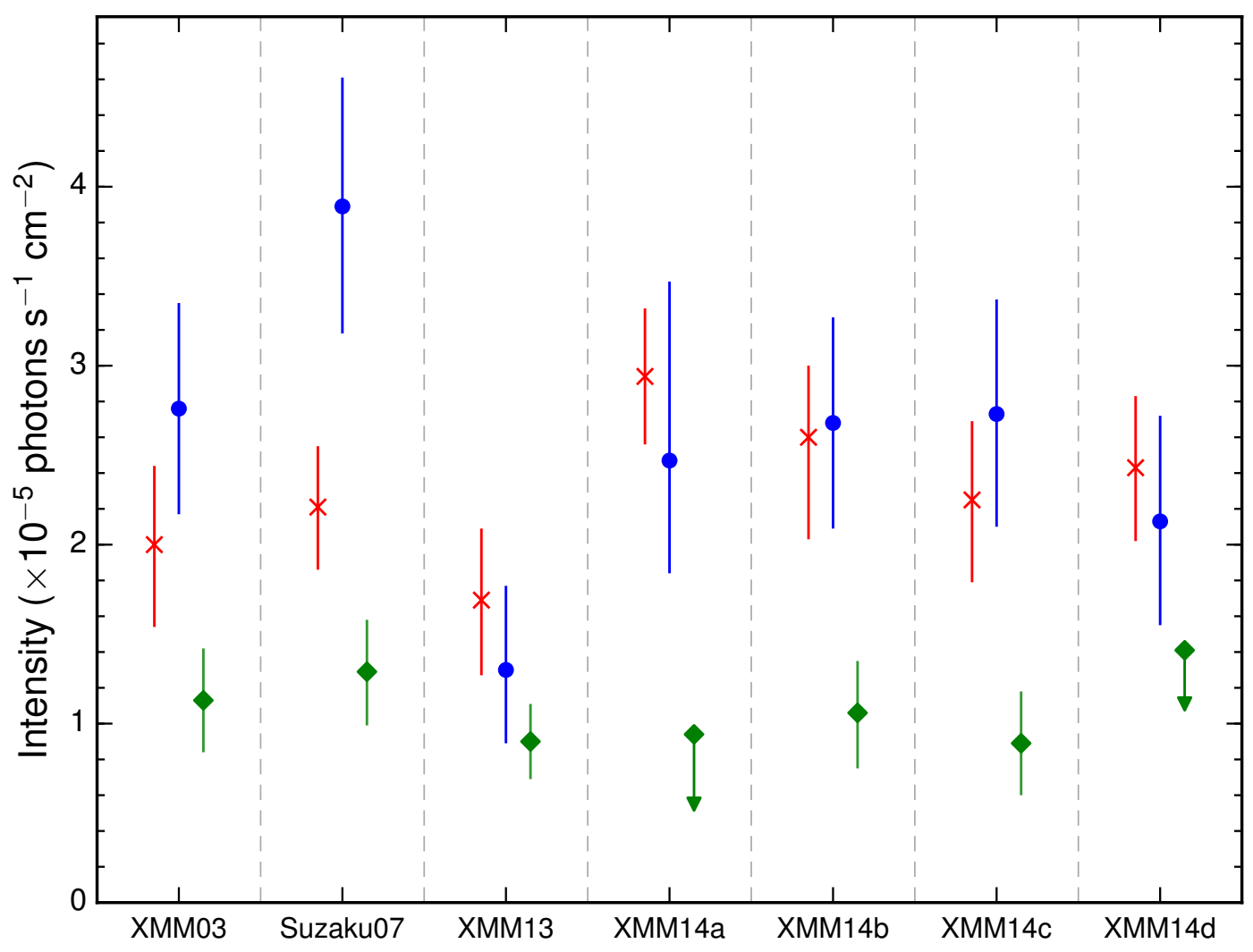

Figure 6. Intensity of the three Fe K $\alpha$ emission features in the 2003, 2013, 2014 XMM-Newton, and 2007 Suzaku spectra of Ark 120: narrow Fe I (6.40 keV, red crosses), tentative Fe XXVI (6.98 keV, green diamonds), and disk line ( $\sim 6.5 \mathrm{keV}$, blue dots). While the two narrow lines have a nearly constant flux, the broad component exhibits a marked drop in 2013 , which is significant at the $5 \sigma$ and $2.5 \sigma$ levels compared to 2007 and $2014 \mathrm{a}$, respectively (the plotted error bars correspond to the $90 \%$ confidence intervals). For simplicity, a cross calibration of 1.00 is assumed between XMM-Newton/pn and Suzaku/XIS. This should be correct within 10\% (Tsujimoto et al. 2011).

not produce a detectable $\mathrm{K} \alpha$ feature. In this view, these regions could yield a featureless pseudo continuum, while a number of hotspots associated with localized magnetic flares further out (e.g., Galeev et al. 1979; Dovčiak et al. 2004a; Goosmann et al. 2007) might dominate the Fe K emission. Indeed, at intermediate (i.e., tens of $r_{\mathrm{g}}$ ) radii, the illumination from a standard (centrally concentrated, compact, and low-height) X-ray corona would be less effective, and an additional source would then be required to sustain the local emissivity. Following on, we modified the relline model assuming an annulus of width $10 r_{\mathrm{g}}$ with gray $(q=0)$ emissivity as the most relevant reflecting surface to the formation of the observed disk line. As could be expected, the fit of the seven spectra is virtually as good as in the original case $\left(\chi^{2} / \nu=1652 / 1575\right)$, with the inner border of each ring commensurate with the usual values for $r_{\text {in }}$ (Table 3). Emission from the entire annulus is only possible if there are at least a few active flares/hotspots at a time. At $60 r_{\mathrm{g}}$, in fact, any structure in Keplerian motion only covers a small fraction $\left(\Delta \phi \sim 20^{\circ}\right)$ of its orbit in the 120 -ks span of a single $X M M-$ Newton observation. In this respect, we switched to the more flexible kyr1line profile (Dovčiak et al. 2004b), which also enables the selection of annular sectors. By freezing either the inner radius or the line's rest energy from the previous fit to control the degeneracy with the central azimuth, we confirmed that the opening angle of each sector is always consistent with being $\Delta \phi \gtrsim 180^{\circ}$, since approaching as well as receding parts of the disk must be involved to account for both the red and the blue wing of the Fe K line.

\subsection{Short timescales (days to hours)}

Depending on the number, radial distance, and lifetime of the putative hotspots, intensity and shape variations in the Fe K emission complex of Ark 120 can occur even on timescales much shorter than one year. In order to appraise any rapid change eluding the analysis of the time-averaged spectra, we applied to the four $2014 X M M-N e w t o n$ sequences an 'excess map' technique, which has proven to be very effective in revealing the possible modulation of redshifted Fe K features in some Seyferts (Iwasawa et al. 2004; Tombesi et al. 2007). We outline here the main steps of this procedure, referring the reader to the previous works for a more detailed description (see also De Marco et al. 2009). We adopted a resolution of $100 \mathrm{eV}$ in energy and $5 \mathrm{ks}$ in time. Since the orbital period at the innermost stable circular 


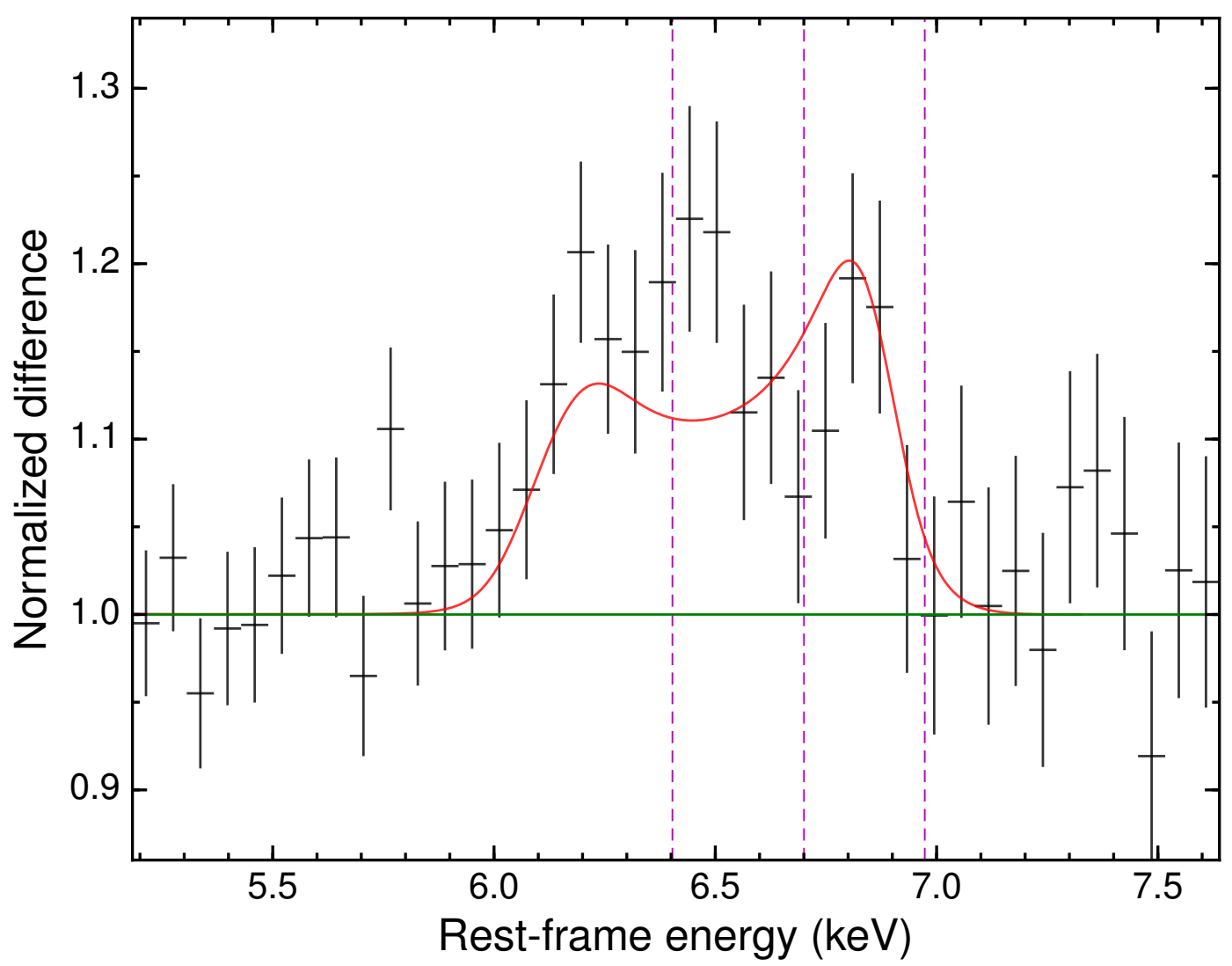

Figure 7. Zoom in on the Fe K emission band of the '2014-2013' difference spectrum, normalized to take into account the different slopes of the hard X-ray continuum in the two epochs and rebinned for clarity. The correction for the relative energy calibration issues has also been applied. It stands out how the emission-line complex is more prominent in the high-flux state. The variations mainly involve the broad component, although an excess is also seen in proximity of the narrow 6.4 -keV core. For comparison, the red curve shows the profile of a disk line with energy of $6.6 \mathrm{keV}$ emitted between 60 and $120 r_{\mathrm{g}}\left(\right.$ for $a^{*}=0$, $i=30^{\circ}$, and $q=3$; note that this is not a fit). The Fe I, Fexxv, and Fe XxvI K $\alpha$ positions are traced for guidance.

orbit of a Schwarzschild black hole with mass of $1.5 \times 10^{8} M_{\odot}$ is about $70 \mathrm{ks}$ (e.g., Bardeen et al. 1972), the latter choice ensures that the reasonable variability timescale in the 'rotating hotspot' scenario is largely oversampled. We then considered the first $115 \mathrm{ks}$ of each observation, neglecting less than $11 \mathrm{ks}$ of the cumulative good-time interval in the rounding. Given the reduced live time of the EPIC/pn Small Window mode, the net exposure of the single $23 \times 4$ slices is $3.5 \mathrm{ks}$. The resulting time-resolved spectra were grouped to a $5 \sigma$ significance per channel, and fitted with a simple power law in the 3-5 plus $7-10 \mathrm{keV}$ range. We worked in the observed frame, and did not correct the energy scale for the calibration problems. In fact, these are not straightforward to tackle, and should affect all the data sets in the same way. The energies of the two narrow lines (6.40 and $6.98 \mathrm{keV}$ at rest) are then shifted to 6.26 and 6.82 $\mathrm{keV}$.

Within the four sequences, a constant trend of the photon index yields a $\chi^{2}$ between 21 and 25 for $\nu=22$ (the typical $90 \%$ uncertainty on the single estimates of $\Gamma$ is \pm 0.07$)$. The average values and $1 \sigma$ dispersions are $\Gamma=1.810( \pm 0.043)$, $\Gamma=1.762( \pm 0.041), \Gamma=1.771( \pm 0.040), \Gamma=1.744( \pm 0.045)$, respectively. Once the continuum is determined, the excess emission map is computed from the residuals in the 100-eV binned spectra, corrected for the detector's collecting area. To verify that all the pixels in the energy-time plane have enough statistics, we recorded the number of counts in the 6.0-6.1 keV bin of each spectral slice. This ranges from 89 to 141, with a mean (median) value of 117 (118). As mentioned, any intensity variation is expected to take place on longer timescales than the sampling time. It is thus possible to suppress the noise between contiguous pixels and emphasize the significant features by applying a low-pass filter (see Iwasawa et al. 2004). We smoothed the image through an elliptical Gaussian kernel with $\left(\sigma_{E}, \sigma_{t}\right)$ $=(1.06,1.27)$ pixels, corresponding to a FWHM of $250 \mathrm{eV} \times 15 \mathrm{ks}$ (i.e., the light-crossing time of $\left.20 r_{\mathrm{g}}\right)$. The final excess emission map is shown in Figure 8, and clearly demonstrates that the Fe K line complex is extremely dynamic. The track of the main, $6.4-\mathrm{keV}$ narrow line is the most persistent, yet it is characterized by intensity fluctuations, especially in the second half of the observation. Highly variable structures are detected both redwards and bluewards, 
with no obvious periodicity nor correlation with each other.

The average noise in the smoothed excess map is $\sim 1 \times 10^{-6}$ photons s $\mathrm{sm}^{-1}$ at the $1 \sigma$ level. All of the hottest blob- and plume-like features in the image contain at least a hundred counts, so their transient nature is expected to be genuine. Quite surprisingly, also the 7-keV narrow line (whose plain, yet mistaken identification from the mere time-averaged spectral analysis would be as Fe XXVI K $\alpha$ from the BLR) erratically brightens and fades on timescales of $\sim 30-50 \mathrm{ks}$, and seems to be one and the same with the blue horn of the disk line. The absence of persistent, prominent Fe XXVI emission has the further advantage of alleviating the apparent discrepancy between the XMM-Newton spectra and the Chandra one, where such a feature is not evident. In this respect, it is worth noting that the actual overlap between the three Chandra/HETG and the four XMM-Newton/EPIC observations is less than 60 ks (Table 1), during which there was only a moderate flaring activity in the blue Fe K excess based on Figure 8. Some BLR-like emission from Fe XXVI cannot be firmly excluded (see Appendix C), but this is impossible to separate from the variable, disk component (and any Fe I K $\beta$ ) with the present data.

Based on a qualitative assessment of Figure 8, we selected three intervals with duration of $30 \mathrm{ks}$, and extracted the relative spectra (with net exposure of $21 \mathrm{ks}$ each) as representative of a quiescent Fe K state (between 10 and $40 \mathrm{ks}$ of the elapsed time), a red flare (75-105 ks), and a blue flare (253-283 ks), respectively. The resulting Fe K profiles are plotted in Figure 9 as ratios over the power-law continuum, for which a common photon index was assumed $(\Delta \Gamma<0.03$ otherwise). During the low-activity phase, only the narrow $(\sigma=40 \mathrm{eV})$ Gaussian line at $6.4 \mathrm{keV}$ is statistically required, with just a hint of asymmetry on the blue side. An extended red wing emerges later on in the first sequence, immediately following and preceding two minor flares in the $6.5-7 \mathrm{keV}$ rest energy band. This blue excess is strongest towards the end of the second observation, when the red wing is back at its faintest, confirming the lack of simultaneity between the two. We note that similar episodes are seen in the last three days of the campaign as well; the spectra are somewhat noisier because the parent events are short-lived and/or less intense, but are always found to be in agreement with those of Figure 9.

To quantify the extent of the intensity variations for the red and the blue excess, we modeled the three spectra including two additional Gaussian lines besides the main $6.4-\mathrm{keV}$ one. This is just a rough approximation, but the current, time-resolved data are not very sensitive to much finer profiles (see below). We fitted a local power law at 4-9 $\mathrm{keV}$ to avoid the subtle continuum complexities (e.g., Figure B1) as well as bins with $<5 \sigma$ significance at the higher end (a 30-eV resolution is still adopted). The photon index and the amplitude of the central feature were tied between the three states, since they do not appear to vary on statistical grounds $\left(\Delta \chi^{2} / \Delta \nu=-2 /-4\right.$ when both are free). The joint fit is very good indeed, with $\chi^{2} / \nu=500 / 485$. The red and the blue line have rest energies of 6.19 and $6.83 \mathrm{keV}$, and widths of $\sim 60$ and $125 \mathrm{eV}$, respectively (Table 5). The intensity of the red Fe K excess increases over the quiescent state and subsequently drops during the blue flare with a $1.7 \sigma$ and $2.1 \sigma$ confidence (see also Appendix D), while the enhancement of the blue excess is significant at the $3.7 \sigma$ level. If the observed variations are due to the differential beaming effects along the orbit of a rotating hotspot on the disk surface, and about one fourth of a Keplerian period $\left(T_{\mathrm{K}}\right)$ is needed for them to be detectable, the characteristic 30-50 ks timescale implies a typical distance from the central black hole of $\sim 10 r_{\mathrm{g}}$. Although the large SMBH mass in Ark 120 and the gaps between the sequences do not facilitate a visual recognition, there does not seem to be any regular modulation of the red and blue features, in either strength or energy. This suggests that the hotspots might not survive for an entire orbit $\left(T_{\mathrm{K}} \simeq 200 \mathrm{ks}\right.$ at $10 r_{\mathrm{g}}$, and $650 \mathrm{ks}$ at $27 r_{\mathrm{g}}$ ), but they should be continuously regenerated. In this scenario, the same red wing and the source of the possible changes seen in the 6.4-keV core (Figure 7) could be explained as the blue horns of Fe K lines arising closer in in the disk.

\section{DISCUSSION}

Ark 120 is an X-ray bright local Seyfert galaxy, prototype of the bare AGN subclass, which is characterized by a clean line of sight to the central source. This allows an unbiased study of some critical components that are commonly found among active galaxies, such as the soft and/or hard X-ray excess, and the possible relativistically blurred iron line. Due to its uniqueness, Ark 120 was simultaneously targeted in a concerted 2014 campaign by XMM-Newton, Chandra/HETG, and NuSTAR. In this work we have taken advantage of the combination of high energy resolution and high effective area afforded by these new data sets in the 6-7 keV band, and revisited the high-quality XMM-Newton and Suzaku archival observations to understand the nature, properties, and variability of the distinctive Fe K emission features.

The interpretation of the Fe K emission complex in Ark 120 is definitely challenging. The same intricate shape implies a blending of different components that are not all readily identifiable, with the only exception of the neutral $\mathrm{Fe} \mathrm{K} \alpha$ at $6.4 \mathrm{keV}$. Moreover, the time-averaged spectral analysis can be rather misleading, if not validated by the supplementary 


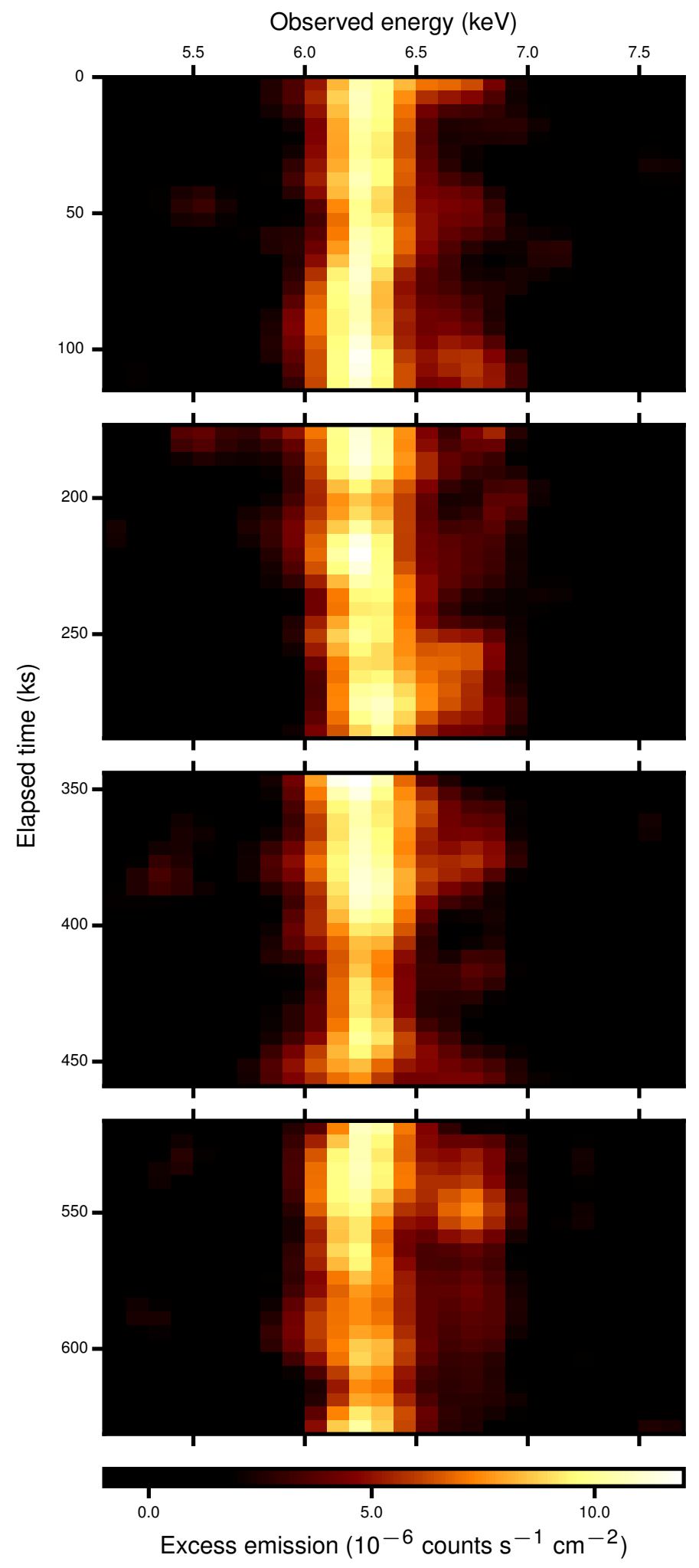

Figure 8. Excess emission map in the Fe K band for the 2014 XMM-Newton observation, with a resolution in the energy-time plane of $100 \mathrm{eV} \times 5 \mathrm{ks}$. The image has been smoothed with an elliptical Gaussian kernel with FWHM of $250 \mathrm{eV} \times 15 \mathrm{ks}$. Energies are in the observed frame, and no gain correction was applied. The two narrow lines from the spectral models are thus centered at 6.26 and $6.82 \mathrm{keV}$. The lower (negative) value of the color scale indicates the $1 \sigma$ noise level, so the main features in the map are expected to be highly significant. As a gauge, considering the effective exposure, an excess of $5 \times 10^{-6}$ photons $\mathrm{s}^{-1} \mathrm{~cm}^{-2}$ corresponds to $\sim 12$ counts. (Note that the time gap between the sequences is not to scale). 


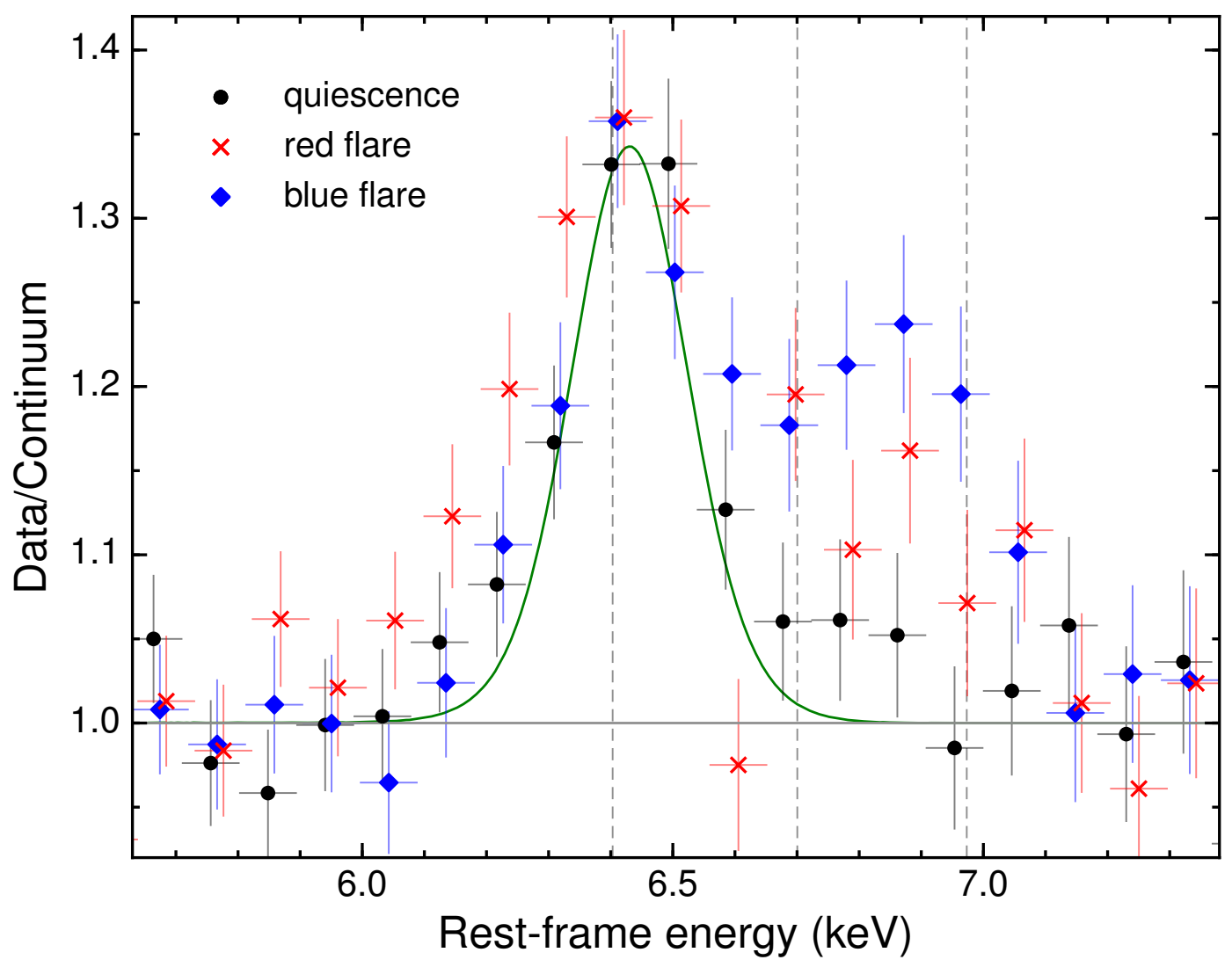

Figure 9. Different profiles of the Fe K emission complex in three 30-ks intervals selected after a visual inspection of the excess emission map in Figure 8, and corresponding to quiescent (dots; 10-40 ks), red flare (crosses; 75-105 ks), and blue flare (diamonds; 253-283 ks) states. Significant changes on short timescales are seen on both sides of the narrow 6.4-keV line (drawn in green), conclusively locating the origin of the broad component(s) from the inner accretion disk. The data are binned by a further factor of three over the adopted $30-\mathrm{eV}$ resolution for visual purposes.

variability study. The presence of a constant, narrow line from Fe XXVI at $6.97 \mathrm{keV}$, for instance, seems to be a mere coincidence, deriving from the combination of some possible iron emission from the BLR (H-like K $\alpha$ and/or neutral $\mathrm{K} \beta$ ) and the blue part of a rapidly variable feature from the disk, when the latter is integrated over sufficiently long (>100 ks) exposures. In this sense, the excess emission map reveals that the broad line itself is likely the random superposition of narrower features from a few, disconnected regions on the disk surface, as opposed to a single circle or annulus. Taken individually, the presumable constituents of the erratic Fe K fluorescent component look neither unresolved nor heavily smeared, and are not subject to large displacements from their usual rest energy $(6.4-7 \mathrm{keV})$. Consequently, they cannot be plainly classified under any of the two flavors of Fe K emission transients that have been found among AGNs, namely narrow and broad, which typically exhibit substantial redshifts. While the sporadic detection of narrow lines at 4-5.5 keV (e.g., Turner et al. 2002; Porquet et al. 2004; Petrucci et al. 2007) might be controversial (see Vaughan \& Uttley 2008 for a critical standpoint), the broad Fe K transients are more intriguing, in particular when the evolution with time of the line's flux and/or centroid can be trailed (e.g., Iwasawa et al. 2004; Turner et al. 2006).

The hotspot picture (e.g., Nayakshin \& Kazanas 2001; Dovčiak et al. 2004a) is widely invoked for both manifestations of variable Fe K features, since it naturally delivers narrow line profiles as well as sizeable intensity and energy changes thanks to the compact emitting region and its orbital motion in a relativistic gravitational field. This is a promising explanation also for the Fe K behavior observed in Ark 120, where the relative contribution of different radial and/or azimuthal segments of the disk surface could drive the variability of the reprocessed Fe K emission on timescales of several hours to a few days. To test the physical applicability of the hotspot model to Ark 120, we fitted once again the quiescent and red/blue flare spectra replacing the two Gaussians for the red and blue Fe K excess with kyr1line components, originating from annular sectors with radial width of $10 r_{\mathrm{g}}$ and flat emissivity $\left(q=0\right.$, plus $\left.a^{*}=0, i=30^{\circ}\right)$. We assumed that the rest energy of both lines is $6.97 \mathrm{keV}$ and, for simplicity, that the same regions of the disk are active in the flaring states. This is unrealistic, of course, but the combinations of line energy (i.e., ionization), distance, 
and azimuth that can give rise to the detected features are manifold, and it is not feasible to discriminate between them all. A self-consistent solution was ensured by linking the angular width of each annular sector (in degrees) to its inner radius through the expression $\Delta \phi=360^{\circ} \Delta t / T_{\mathrm{K}}(r)=1.16 M_{7}^{-1}\left(r_{\mathrm{in}}+0.5 \Delta r\right)^{-1.5} \Delta t$ (adapted from Bardeen et al. 1972), where $M_{7}=15$ is the black hole mass in units of $10^{7} M_{\odot}, \Delta r=10 r_{\mathrm{g}}$, and $\Delta t=30 \mathrm{ks}$. While a smaller $\Delta r$ would be probably more appropriate for an isolated hotspot, flares are thought to come in avalanches (Poutanen \& Fabian 1999), hence a wider area might be coherently illuminated. We also note that larger structures would be disrupted more quickly by differential rotation, in agreement with the short-lived events observed here.

The resulting fit statistic is exactly the same of the phenomenological attempt above $\left(\chi^{2} / \nu=500 / 485\right)$. Indeed, as the range of angles involved is limited $\left(\Delta \phi=11^{\circ}\right.$ for the red horn and $25^{\circ}$ for the blue one), the emitted lines are narrow, and lose any asymmetry after the convolution with the detector's response, becoming in practice indistinguishable from simple Gaussian profiles (Figure 10). We chose a framework where positive angular velocity corresponds to counterclockwise rotation, and $\phi=0^{\circ}$ to the maximal Doppler blueshift for matter moving towards the observer. The red feature then stems from a sector delimited by $r \simeq 30-40 r_{\mathrm{g}}$ and $\phi \simeq 217-228^{\circ}$, while the boundaries for the blue one are $r \simeq 15-25 r_{\mathrm{g}}$ and $\phi \simeq 40-65^{\circ}$. The significance of their variability is confirmed at the $2.2 \sigma$ and $3.6 \sigma$ levels. Once the uncertainties on the best-fit parameters are considered (Table 5), it is explicit that the two active regions above, responsible for the red (6.0-6.3 keV) and the blue (6.5-7.0 keV) Fe K excess, are widely separated and respectively confined to the receding and approaching parts of the disk. Although there is some overlap in the range of distances they are allowed to lie at, between $\sim 20-30 r_{\mathrm{g}}$, in the absence of clear periodic patterns in the excess emission map a single hotspot appears unlikely. On the other hand, some arc-like structures (like at $t \sim 200-230$ ks; Figure 8) are strongly suggestive of orbital motions. Trying to describe the whole variability of the Fe K features in these terms, however, goes beyond the scope of the present work.

An energy reservoir formed by an ensemble of buoyant magnetic loops/blobs has been recurrently proposed to explain the X-ray spectra and light curves of AGNs (e.g., Galeev et al. 1979; Haardt et al. 1994; Merloni \& Fabian 2001; Czerny et al. 2004). The stored energy would be released through field reconnection events, which sustain the energetic stability of the corona and, in principle, can induce appreciable flares in the primary X-ray emission. Part of this radiation impinges on the disk below, and is reflected off the surface. Based on the X-ray fractional variability and the log-normal flux distribution of AGNs, the number $\mathcal{N}_{\text {flare }}$ of uncorrelated active flare/hotspot regions present at any given time must be limited (Uttley et al. 2005). In Ark 120, the amplitude of the intensity fluctuations and the same occurrence of quasi-quiescent $\mathrm{Fe} K$ states are consistent with $\mathcal{N}_{\text {flare }}$ being no more than a few. It is not surprising that this coronal component is not the main source of primary X-ray emission, then. We can guess the intrinsic, cumulative luminosity of the hotspots by extrapolating the reflection component from the relxill model of Section 3.4. Over the $0.1-100 \mathrm{keV}$ band, this is $5-6 \times 10^{43} \mathrm{erg} \mathrm{s}^{-1}$ in the four sequences of the 2014 campaign. For an average reflection fraction $\mathcal{R} \simeq 0.25$ (Table 4), we can argue that about one fourth of the power-law continuum emerges from the flares, bringing a further $8-9 \times 10^{43} \mathrm{erg} \mathrm{s}^{-1}$. In the most favorable conditions of zero time lag (i.e., $h \ll r$ ) and isotropic emission (which is not so obvious in the local frame), with just $\mathcal{N}_{\text {flare }} \sim 3-5$ the single flare/hotspot would thus contribute to $\sim 5 \%$ only of the total $0.1-100 \mathrm{keV}$ luminosity of Ark $120\left(6.5 \times 10^{44} \mathrm{erg} \mathrm{s}^{-1}\right.$; Porquet et al. 2016), and still less than $10 \%$ restricting to the $2-10 \mathrm{keV}$ band. The fact that no sudden spike is seen in the light curves is therefore plausible. For the same reasons, even the strongest fluorescent lines in the soft X-rays (like O VIII Ly $\alpha$ at $0.654 \mathrm{keV}$ ) should be completely missed in the EPIC/pn spectra, while the low RGS effective area prevents any attempt of time-resolved analysis. Interestingly, in the model by Haardt et al. (1994) the most luminous coronal blobs are located at $r=24 r_{\mathrm{g}}$, and for the black hole mass and Eddington rate of Ark 120 (roughly 0.1) they should emit a few $\times 10^{43} \mathrm{erg} \mathrm{s}^{-1}$.

In their recent study on the geometry of AGN coronae, Wilkins \& Gallo (2015) have suggested that the very detection of X-ray reflection signatures from the surroundings of the innermost stable circular orbit requires a patchy coronal structure. While a number of clumps could appear as a continuous, extended medium in long exposures, the reflected spectrum would still be exempt from heavy Comptonization effects by leaking through the voids. Our analysis of the Fe K transients in Ark 120 hints at a hybrid situation, where the X-ray corona is smooth in the center and patchy towards the edges. This would simultaneously justify the apparent lack of reflection from the inner disk (except, perhaps, for the 2007 Suzaku observation) and the short-term Fe K variability. It might be appealing to associate the characteristic radius of the transitional region where the observed features arise with a change of the physical conditions within the accretion flow. A popular Comptonization model like optxagn (Done et al. 2012), for instance, adopts the size of the corona as a key dividing line between the outer and the inner disk, setting the energy balance between thermal emission, soft X-ray excess and hard X-ray power law. Matt et al. (2014) made use of optxagn in their broadband fits to the $2013 \mathrm{XMM-Newton/NuSTAR}$ spectra of Ark 120, and obtained a coronal extent of $\sim 10-30 r_{\mathrm{g}}$, 
Table 5. Fe K excess features in the flare/hotspot scenario.

\begin{tabular}{|c|c|c|c|c|c|}
\hline \multicolumn{3}{|c|}{ zgauss } & & \multicolumn{2}{|c|}{ kyr1line } \\
\hline & red & blue & & red & blue \\
\hline$E$ & $6.19_{-0.07}^{+0.07}$ & $6.83_{-0.04}^{+0.05}$ & $r_{\text {in }}$ & $30_{-14}^{+12}$ & $15_{-4}^{+6}$ \\
\hline$\sigma$ & $<169$ & $125_{-46}^{+57}$ & $\phi$ & $217_{-127 p}^{+33}$ & $40_{-13}^{+13}$ \\
\hline$I_{\mathrm{f}}$ & $1.66_{-0.76}^{+1.05}$ & $3.01_{-0.80}^{+0.93}$ & $I_{\mathrm{f}}$ & $1.58_{-0.67}^{+1.15}$ & $2.97_{-0.83}^{+1.02}$ \\
\hline$I_{\mathrm{q}}$ & $<1.02$ & $<1.27$ & $I_{\mathrm{q}}$ & $<0.98$ & $<1.25$ \\
\hline
\end{tabular}

Notes. In both the Gaussian and disk line cases, the continuum photon index is $\Gamma=1.83( \pm 0.03)$, and the energy of the narrow $\mathrm{K} \alpha$ is $E=6.42( \pm 0.02)$. The intensities of the variable lines during the flaring $\left(I_{\mathrm{f}}\right)$ and quiescent $\left(I_{\mathrm{q}}\right)$ states are in units of $10^{-5}$ photons $\mathrm{s}^{-1} \mathrm{~cm}^{-2}$. The rest energy of each kyr1line component is fixed at $6.97 \mathrm{keV}$ (see the text for the other assumptions of the model). The lower azimuth of the emitting sector $(\phi)$ is measured in degrees from the position of maximal approaching speed. Errors are given at the $90 \%$ confidence level.

depending on the chosen black hole spin. While remarkably similar to the inferred location of the hotspots, the latter can still be seen as a convenient parameterization. However, the existence of a critical distance scale is also envisaged on theoretical grounds. Indeed, for Eddington rate values as the one of Ark 120 a standard geometrically thin, optically thick accretion disk (Shakura \& Sunyaev 1973) should invariably undergo a transition from gas to radiation pressure dominated below a certain radius, unless all of the accretion power is dissipated in the corona (Svensson \& Zdziarski 1994). Structure and properties of the X-ray source could then be radically different through this physical boundary, with the fragmentation of the corona into filaments/clumps at its outskirts.

Alternatively, the cause of $\mathrm{Fe} \mathrm{K}$ variability can be fully inherent to the disk. In fact, the radiation pressure dominated zone was soon realized to be unstable against thermal and viscous perturbations (Lightman \& Eardley 1974; Shakura \& Sunyaev 1976). Other dynamical instabilities follow in magnetized disks, leading to widespread turbulence (see Balbus \& Hawley 1998 for a comprehensive review) and large density gradients (e.g., Begelman 2001). The ensuing inhomogeneity significantly alters the spectral features reprocessed from the disk with respect to the usual case of a flat, uniform slab. The reflection component would bear the different signs of more rarefied versus compact layers, and even moderate density variations can deeply affect the intensity of the Fe K emission without any correlation with the illuminating continuum (Ballantyne et al. 2004, 2005). The continual succession of clumps and voids at any given location and the related emissivity changes are then a potential explanation for the Fe $\mathrm{K}$ variability. Relativistic beaming induced by turbulence can also be important (Armitage \& Reynolds 2003). Among the various clumping instabilities, of particular relevance to Ark 120 could be the so-called 'photon bubbles' (Gammie 1998), which, differently from most of the other perturbations, can develop on timescales much shorter than the orbital period (Blaes \& Socrates 2003).

A theoretical effort to predict the empirical differences between orbiting hotspots, coronal clumps, and disk instabilities could be worthwhile in the future. Regardless of the actual mechanism responsible for the transient Fe K emission observed in Ark 120, however, our findings prove that the environment pervaded by the inner accretion flow is almost certainly highly dynamic and/or inhomogeneous. If these conditions, and the underlying physical processes, are established to be common among AGNs, there would also be several compelling implications on the origin of the broad Fe K lines detected in many sources. In fact, some of these profiles could result from the integrated contribution of individual, disconnected regions, especially at lower $\left(10^{6}-10^{7} M_{\odot}\right)$ black hole masses, hence proportionally shorter orbital timescales.

\section{CONCLUSIONS}

We have presented the analysis of the composite emission spectrum due to iron K-shell fluorescence in the local Seyfert galaxy Ark 120, the nearest and brightest example of a bare AGN, based on a large 2014 campaign consisting of simultaneous XMM-Newton, Chandra/HETG, and NuSTAR observations. Overall, Ark 120 displays a broad and irregular emission-line complex in the 6-7 keV energy range, where the main feature corresponds to the $\mathrm{K} \alpha$ transition in neutral iron around $6.4 \mathrm{keV}$. Its profile is well resolved by the Chandra grating to a FWHM of $\sim 4700 \mathrm{~km} \mathrm{~s}^{-1}$, consistent with the typical velocity broadening of the optical broad-line region. The narrow Fe I K $\alpha$ accounts for less than one half of the total line emission over this band, though. The residuals take the shape of a red wing down to $\sim 6 \mathrm{keV}$ and of a blue plateau across $6.7 \mathrm{keV}$, connecting to a second, apparent feature centered just below $7 \mathrm{keV}$. Energy, equivalent width, and variability rule out an interpretation of the red excess as the Compton shoulder of the main, 6.4-keV K $\alpha$ line. The wing's strength reached a maximum during a 2007 Suzaku observation, dropped by a 


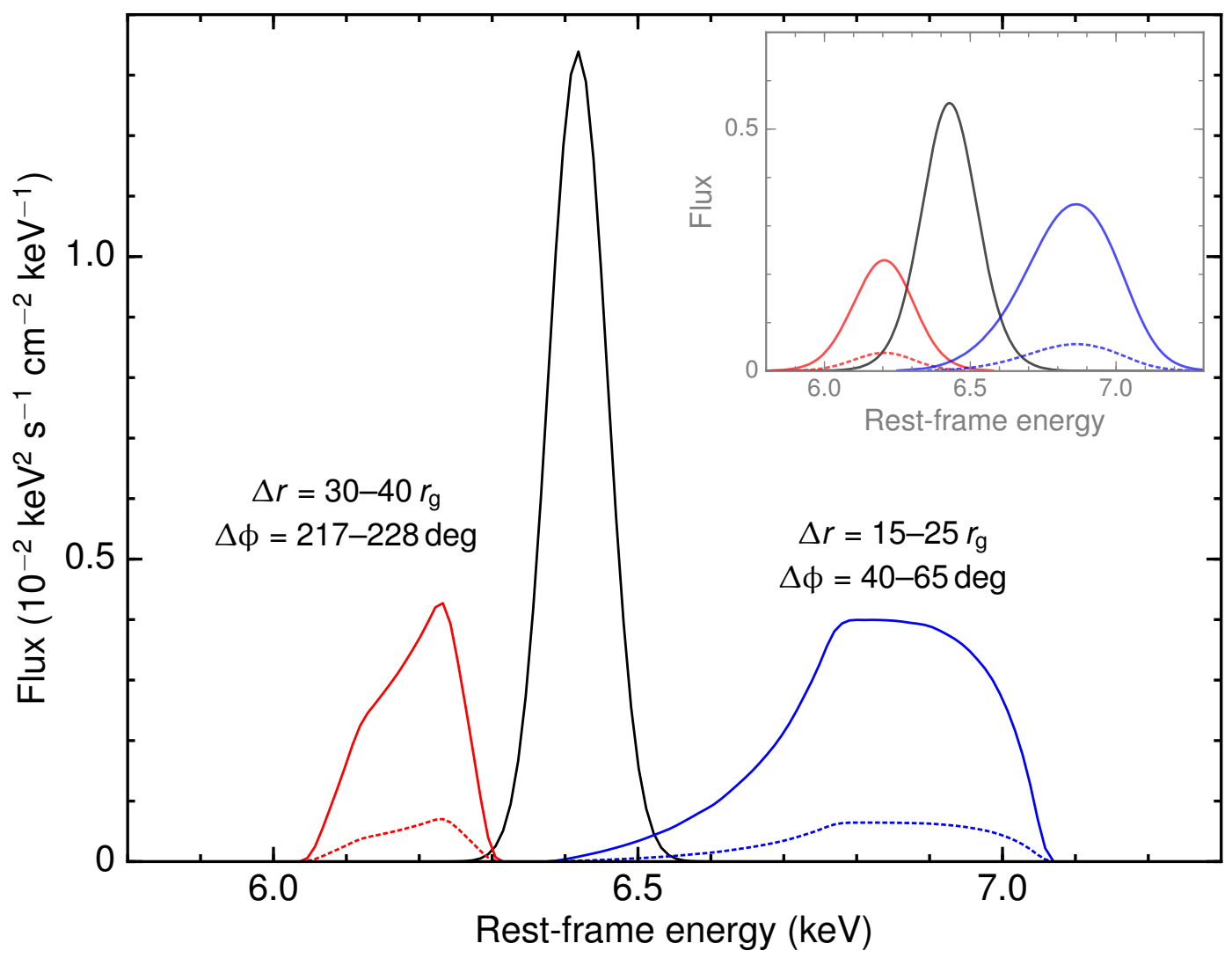

Figure 10. Fe K emission components in the quiescent, red flare, and blue flare spectral states for the hotspot model. The narrow, BLR-like K $\alpha$ from neutral iron at $6.4 \mathrm{keV}$ (in black) is constant, while the highest (solid) and lowest (dashed) intensities are shown for the red and the blue transient features. The radial and azimuthal coordinates of the relative active regions are also printed, for $a^{*}=0, i=30^{\circ}$, and a rest energy of the line of $6.97 \mathrm{keV}$ ( $\phi=0^{\circ}$ corresponds to the maximal approaching speed). The profiles are computed assuming an ideal response with linear resolution of $10 \mathrm{eV}$, while the inset shows their smearing after convolution with the EPIC/pn response (note that the vertical scale is compressed by a factor of two). The intrinsic asymmetry is almost completely lost at CCD resolution.

factor of $\sim 2-3$ following a low-flux state of the X-ray source in 2013, and then recovered one year later. This behavior suggests the presence of a Fe K reflection component from the accretion disk. Indeed, a model including two narrow $(\sigma \simeq 40 \mathrm{eV})$ lines at 6.40 and $6.98 \mathrm{keV}$ plus a mildly distorted relativistic disk line successfully reproduces the entire FeK emission complex in Ark 120 over the different epochs. From the time-averaged spectra alone the intensity of the former pair would seem approximately constant, as might be expected for BLR gas, only sensitive to the average continuum over long timescales. The $7-\mathrm{keV}$ narrow feature would thus be (mistakenly) identified with the Fe XXVI $\mathrm{K} \alpha$, possibly blended with the neutral $\mathrm{K} \beta$. Some additional fluctuations around $6.4-6.5 \mathrm{keV}$ could be ascribed to the elusive broad component, which is responsible for most of the short-term spectral changes.

The disk-line parameters point to an origin in a moderate relativistic regime, at a few/several tens of gravitational radii of distance from the central black hole. Rather than a disk truncation, this likely implies that the main Xray corona, if extended, smooths out any reflection from the inner disk. Diffuse flares associated with magnetic reconnection events might be involved instead to efficiently illuminate the line-emitting regions further out. Such a conjecture is supported by the FeK excess emission map obtained from the 2014 XMM-Newton monitoring, which covered four consecutive satellite orbits for a span of 7.5 days. This shows that both the red wing and the bulk of the 6.5-7 keV excess (encompassing also the tentative, BLR-like Fe XxvI line) undergo significant intensity variations in about $\sim 30-50 \mathrm{ks}$ (i.e., 10-15 hours), and that they are substantially uncorrelated with each other. The broad Fe K emission feature detected in Ark 120 could then be the superposition of several different peaks arising from short-lived, yet continuously generated orbiting hotspots on the accretion disk surface. Perhaps similar observable effects could be produced also as a result of clumpiness in the corona and/or density inhomogeneities in the disk, provided that these are highly dynamic.

Future X-ray observatories like Athena (Nandra et al. 2013) will afford the higher energy resolution and larger effective area in the 6-7 keV band that are needed to reveal any fine structures in the observed Fe $\mathrm{K}$ profile and to 
perform a proper time-resolved spectral analysis, so disentangling the various BLR and disk contributions and tracing the evolution of the putative hotspots. Thanks to its bare nature, Ark 120 stands out as the most promising source to study the accretion disk/X-ray corona system in AGNs, and its possible flaring, transient components.

The authors would like to thank the anonymous referee for their useful comments that helped improving the clarity of this paper. EN is supported by the UK Science and Technology Facilities Council under grant ST/M001040/1. DP acknowledges financial support from the French Programme National Hautes Energies (PNHE) and the EU 7th Framework Programme FP7 (2013-2017) under grant agreement number 312789. JNR acknowledges support from Chandra grant number GO4-15092X and NASA grant NNX15AF12G. AL acknowledges support from the UK STFC. The results presented in this paper are based on data obtained with the Chandra X-ray Observatory; XMM-Newton, an ESA science mission with instruments and contributions directly funded by ESA member states and NASA; and Suzaku, a collaborative mission between the space agency of Japan (JAXA) and NASA. We have made use of software provided by the Chandra X-ray Center (CXC) in the application package CIAO. The figures were generated using matplotlib (Hunter 2007), a PYTHON library for publication of quality graphics.

\section{APPENDIX}

\section{A. GAIN CORRECTION}

Even before embarking on a proper spectral fitting, we noticed that, in each of the four 2014 XMM-Newton data sets, the stronger among the K-shell emission features is peaked at $6.45-6.47 \mathrm{keV}$. Rather than signifying a mild ionization state of the gas (e.g., Fe XIX; Kallman et al. 2004), this is due instead to a known inaccuracy in the calibration of the EPIC/pn energy scale for some of the most recent XMM-Newton observations (as also discussed in Marinucci et al. 2014), and the actual line identification is with the neutral $\mathrm{K} \alpha$. Indeed, in the simultaneous Chandra data, its centroid falls at $E=6.416_{-0.017}^{+0.016} \mathrm{keV}$ (Section 3.1). Overall, this $\sim 50-\mathrm{eV}$ shift in the iron-K energy range is not the most conspicuous shortcoming. Regardless of the model adopted for the continuum, a clear residual structure is left around $\sim 2.2-2.3 \mathrm{keV}$, in correspondence with the gold absorption edge in the detector's effective area. While not strictly relevant to the analysis presented in this paper, this feature was very useful to mitigate the calibration problems. As a first approximation, we used the gain function within XSPEC, which modifies the energy response according to the expression $\mathcal{E}=E / a+b$ (in $\mathrm{keV}$ ). We then fitted the average $2014 m$ spectrum (obtained by merging the four observations) over the 1-10 keV band, by means of a phenomenological double power-law model, plus the required emission lines. The best slope and offset to fix the gain around the Au-M edge are $a \simeq 1.009$ and $b \simeq 0.004$ $\mathrm{keV}$, respectively. As a by-product, these parameters also return sensible values of the iron-K energy scale (with the main line now falling at $E \simeq 6.41 \pm 0.01 \mathrm{keV}$; Section 3.2), and were thus assumed in all of the analysis. To a lesser degree, even the 2013 spectrum appears to be affected by some gain issues. Its response was then improved through a similar procedure, with $a=1.004$ and $b=-0.002 \mathrm{keV}$. All the line energies reported in the paper for both the 2013 and the 2014 XMM-Newton observations are gain-corrected. Remarkably, as the 2003 EPIC/pn data are confidently exempt from calibration problems, the centroids of the two narrow lines in this epoch (Table 3) corroborate the validity of the adopted gain correction for the most recent spectra, independently of Chandra/HEG.

\section{B. CONTINUUM MODELING}

As long as the evaluation of the emission components' parameters is the foremost intention of our analysis, we verified that the exact continuum shape has no practical consequence. Indeed, thanks to the lack of any X-ray absorption in Ark 120, the 3-10 keV continuum shows little complexity, and can be approximated with no loss of accuracy with a simple power law. For instance, here we consider the relline model described in Section 4.1. By removing the three emission lines, and neglecting the observed 5-7 keV energy range, the fit statistic for the 3-5 plus 7-10 keV power-law continuum is $\chi^{2} / \nu=1232 / 1134$. Any residual spectral curvature can then be estimated by introducing a fictitious partial covering absorber and refitting. The improvement $\left(\chi^{2} / \nu=1191 / 1120\right)$ is similar to the one achieved through the relxill model (Section 3.4; $\chi^{2} / \nu=1196 / 1113$ over the same range), and is mainly driven by the width of the continuum window (see also footnote 3 ). Even allowing for a slight curvature, the lines' properties would be completely unaffected. The relline fit residuals are shown in Figure B1, as an illustration of the good continuum reproducibility through a power law. 


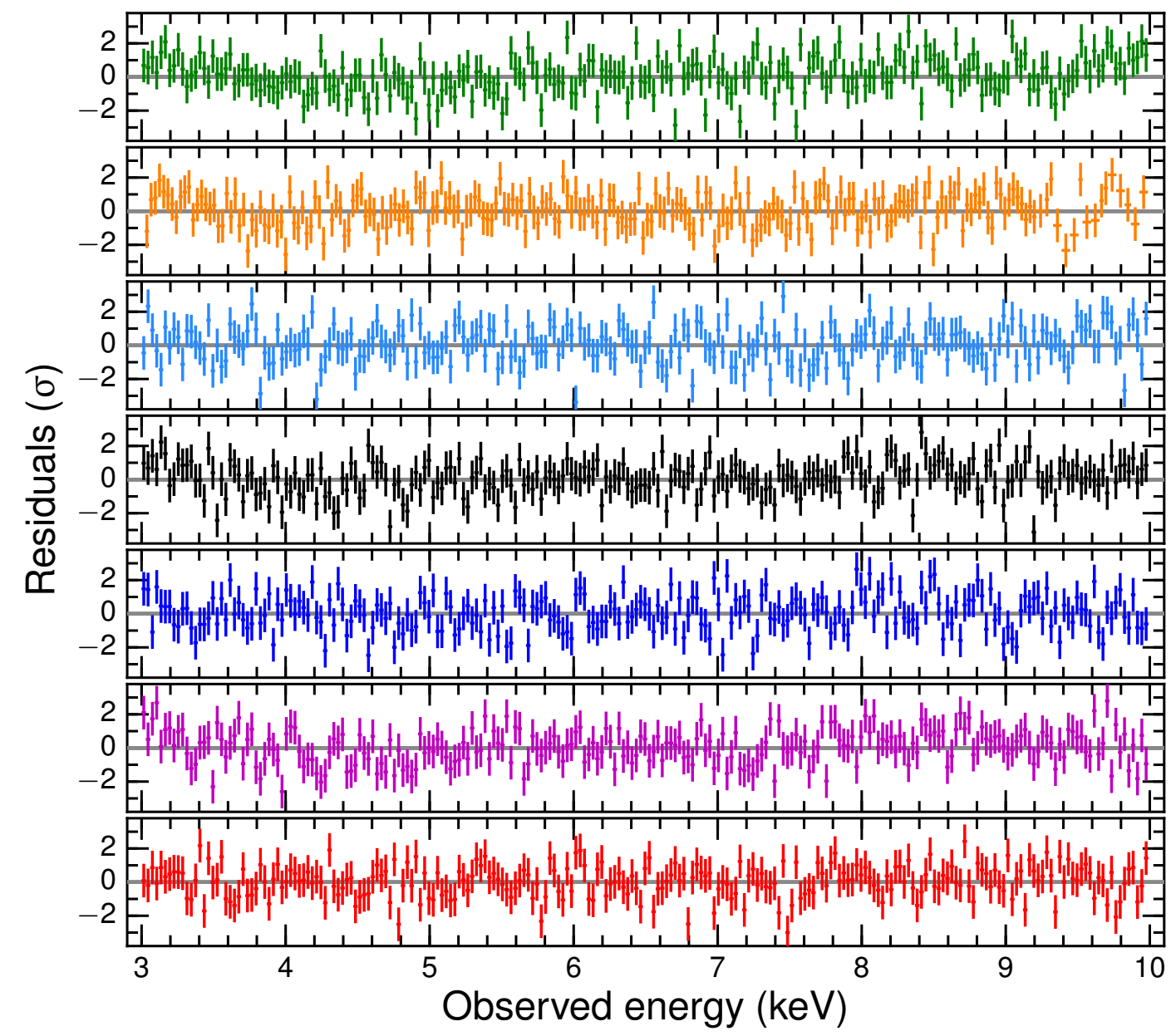

Figure B1. Best-fit residuals (in units of $\sigma$; error bars have size 1) for the relline model applied to all the 3-10 keV spectra of Ark 120 (Section 4.1), showing that the continuum can be always well reproduced by a simple power law. In chronological order, from top to bottom: XMM-Newton 2003, Suzaku 2007, XMM-Newton 2013, 2014a, 2014b, 2014c, and 2014d (the same color code of Figures 3 and 5 is used).

\section{NARROW EMISSION FROM IONIZED IRON}

Given the baffling variability properties of the apparent narrow feature at $\sim 7-\mathrm{keV}$, a logical possibility to check is whether the $\mathrm{K} \beta$ line from neutral iron partly contributes to the bluest emission structures. We then introduced another Gaussian in the relline model, fixing its energy to $7.06 \mathrm{keV}$ and its intensity to 0.15 times the $6.4-\mathrm{keV} \mathrm{K} \alpha$ one (e.g., Molendi et al. 2003). The subsequent fit of the merged 2014m XMM-Newton spectrum proved that the new line is not strongly required statistically $\left(\Delta \chi^{2}=-4\right)$, and that its main effect is to push the neighboring, purported Fexxvi K $\alpha$ redwards to $6.92 \pm 0.03 \mathrm{keV}$. Since the two features appear to some extent mutually exclusive, we then tried to drop the latter and let the relativistic line compensate for the lack of any narrow emission from ionized iron species. As a result, the relline centroid moves to $6.68_{-0.03}^{+0.02} \mathrm{keV}$ (compatible with Fe XXV K $\alpha$ at rest), yet the red wing is not perfectly reproduced. This is reflected by the overall change in the goodness of fit, with $\Delta \chi^{2} / \Delta \nu=16 / 2$ (for an $F$-test chance probability of $1.1 \times 10^{-3}$ ). We conclude that the 7 -keV excess, besides the variable component from the disk, possibly contains also a blend of narrow Fe XxVI K $\alpha$ and Fe I K $\beta$, whose relative and total intensities are very hard to determine. The presence of Fe XXVI is supported by some conspicuous residuals at $E=8.20_{-0.05}^{+0.06}$ and $8.72_{-0.07}^{+0.06} \mathrm{keV}$ in $2014 \mathrm{~m}$, consistent with the higher-order $\mathrm{K} \beta$ and $\mathrm{K} \gamma$ transitions. When fixed at 8.25 and $8.70 \mathrm{keV}$ with the same FWHM of the other narrow lines, these two features bring an improvement of $\Delta \chi^{2}=14.7$ and 15.5 against the loss of two d.o.f., lowering the fit statistic of $2014 m$ to $\chi^{2} / \nu=231 / 222$. Due to the huge difference in ionization potential, it is definitely unlikely for neutral and H-like iron to be co-spatial, thus requiring a substantial BLR stratification. 


\section{VARIABILITY SIGNIFICANCE}

In order to quantify the preference of a 'hotspot' scenario over a standard 'disk line' one, involving two narrow, BLRlike emission lines plus a coherent, broad feature, we also fitted the quiescent, red flare, and blue flare states (Figure 9) with the relline model adopted for the time-averaged spectra. The intensity of the three lines was assumed to be constant with time. This returns a $\chi^{2} / \nu$ of $529 / 490$, clearly worse than the outcome of the hotspot model (500/485; with a pair of variable Gaussian/kyr1line components besides the $6.4-\mathrm{keV} \mathrm{K} \alpha$ ), yet still broadly acceptable. With respect to the $2014 \mathrm{~m}$ best fit (Table 3), there are two main differences: the 7-keV narrow line is shifted to $E=7.03$ $\mathrm{keV}$, although the centroid energy is virtually unconstrained due to the sizeable intensity drop $(\mathrm{EW} \simeq 10 \mathrm{eV})$; and the disc line is now found at $E_{\mathrm{R}}=6.63_{-0.32}^{+0.17} \mathrm{keV}$ (with $r_{\text {in }} \sim 50 r_{\mathrm{g}}$ ). Overall, these findings are thus consistent with the case discussed in Appendix $\mathrm{C}$, where the neutral $\mathrm{K} \beta$ takes the place of the Fe XXVI K $\alpha$. As the two models are non-nested, a proper statistical comparision can be obtained by means of the corrected Akaike Information Criterion (AIC; Akaike 1974) and/or of the Bayesian Information Criterion (BIC; Schwarz 1978). For $k$ free parameters, $N$ data points, and $k^{\prime}=k N /(N-k-1)$, these are defined as:

$$
\mathrm{AIC}=\chi^{2}+2 k^{\prime}, \quad \mathrm{BIC}=\chi^{2}+k \ln N .
$$

The correction term in AIC accounts for the finiteness of the sample. From the single AIC values, the Akaike weight of each model can be computed as $\mathcal{W}_{\mathrm{AIC}} \propto \exp \left(-\Delta_{\mathrm{AIC}} / 2\right)$. For the 'disk line' case, it is $\mathcal{W}_{\mathrm{AIC}} \simeq 1.6 \times 10^{-4}$, so indicating that the 'hotspot' picture is strongly preferred by the data. Conversely, BIC penalizes much more heavily than AIC the use of unnecessary free parameters (on aggregate, 16 in the latter model against 11 in the former), and would still marginally favor the 'disk line' scenario in spite of the worse $\chi^{2}$. The difference $\Delta_{\mathrm{BIC}}=2$, however, is too small to be conclusive: the Schwarz weights, equivalent to the Akaike ones, are 0.628 (disk line) and 0.372 (hotspot).

As the AIC and BIC results are contradictory, and the merits of one method over the other in any specific situation are still matter of debate (e.g. Liddle 2004, and references therein), we proceeded to further assess the true significance of Fe K variability via Monte Carlo simulations. The constant relline model, as evaluated above on the three distinctive spectral states extracted from the excess emission map, represents our null hypothesis. We first ran a test simulation, generating three spectra (and relative background) with the same exposure $(21 \mathrm{ks})$ and response files of the quiescent, red flare, and blue flare states. These were jointly fitted to derive a modified null hypothesis, which incorporates the uncertainties due to photon noise (see also Miniutti \& Fabian 2006). With this refined model, we then performed the actual simulation. The output spectra were fitted twice, with the (constant) relline model and the (variable) hotspot one. The $\Delta \chi^{2}$ was recorded, and all the steps were repeated 1000 times. The following $\Delta \chi^{2}$ distribution can be compared to the statistical improvement characterizing the observed data. In no case out of 1000 is $\Delta \chi^{2}<-29.0$, the largest deviation from the null-hypothesis fit being $\Delta \chi^{2}=-27.6$ (Figure D2). With a false positive rate of less than $10^{-3}$, it seems highly unlikely that the red and blue Fe K transient features are caused by noise in a constant disk-line profile. Most of all, the lack of variability is hard to reconcile with the quiescent phase. With this in mind, we finally checked the significance of the changes experienced by the red Fe K excess. For simplicity, we only examined the quiescent and red flare states. Forcing the intensity of the red horn to be the same in both spectra, the fit deteriorates by $\Delta \chi^{2}=4.8$. We therefore ran 1000 simulations according to the procedure described above, with a null hypothesis of no variability. Each pair of fake data sets were then fitted with common and distinct normalizations of the Gaussian profile at $\sim 6.2 \mathrm{keV}$. The chance of obtaining a spurious improvement with $\Delta \chi^{2}<-4.8$ is $37 / 1000$ (Figure D2). The variability of the red feature is then significant at the $96.3 \%$ level, in agreement with both the $F$-test probability $(96.9 \%)$ and the difference between the line's normalizations in the two spectral states $(\sim 2 \sigma$ confidence; Section 5$)$. We note that this is a largely conservative figure from a general perspective, since the red excess is fainter than the blue one (maximum EW of $\sim 40 \mathrm{eV}$ against $\sim 90 \mathrm{eV}$ ), and the upper limit to its intensity is even lower in the blue flare state (with $\Delta \chi^{2}=6.6$ for constant amplitude).

\section{REFERENCES}

Akaike, H. 1974, IEEE Transactions on Automatic Control, 19, 716

Armitage, P. J., \& Reynolds, C. S. 2003, MNRAS, 341, 1041

Ballantyne, D. R., Turner, N. J., \& Blaes, O. M. 2004, ApJ, 603, 436

Ballantyne, D. R., Turner, N. J., \& Young, A. J. 2005, ApJ, 619, 1028
Bardeen, J. M., Press, W. H., \& Teukolsky, S. A. 1972, ApJ, 178, 347

Baumgartner, W. H., Tueller, J., Markwardt, C. B., et al. 2013, ApJS, 207, 19

Begelman, M. C. 2001, ApJ, 551, 897

Bianchi, S., Guainazzi, M., Matt, G., \& Fonseca Bonilla, N. 2007, A\&A, 467, L19

Blaes, O., \& Socrates, A. 2003, ApJ, 596, 509 


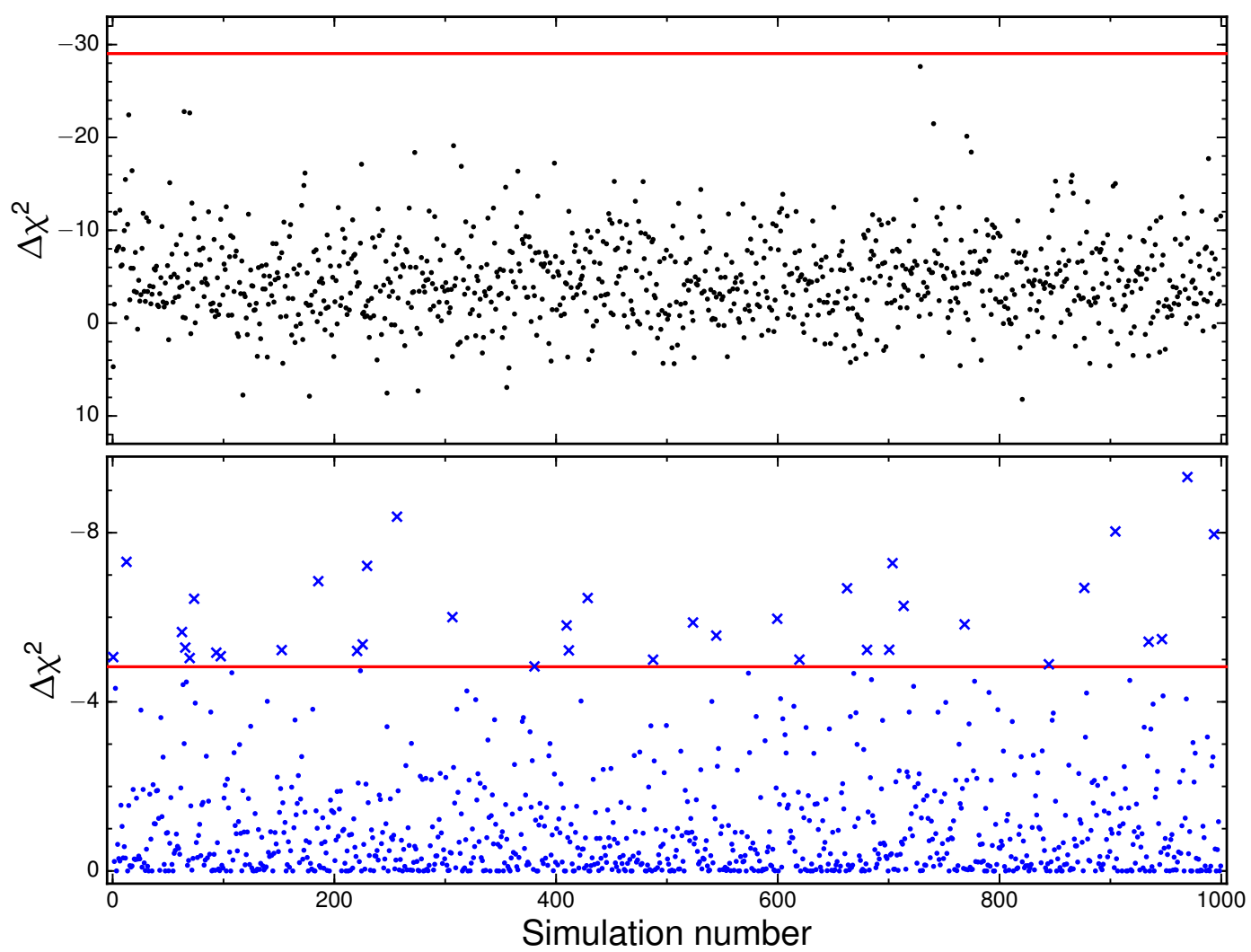

Figure D2. Distributions of $\Delta \chi^{2}$ with respect to the null-hypothesis fits obtained from Monte Carlo simulations. Top panel: the three fake spectra (with same exposure, background, and flux of the real quiescent, red flare, and blue flare states) are jointly fitted with both constant 'disk line' and variable 'hotspot' models. In a single case the statistical improvement is close to, yet still lower than the one found in the real data $\left(\Delta \chi^{2}=-29.0\right.$, red solid line), thus confirming the high significance of the short-term Fe K variability. Bottom panel: the simulated quiescent and red flare spectra are fitted with constant (null hypothesis) and variable intensity of the red Fe K excess. The number of false positives (37/1000) sets a conservative confidence level for the observed variability of the individual transients to $96.3 \%$ (see text).

Brandt, W. N., Fabian, A. C., Nandra, K., \& Tsuruta, S. 1993, MNRAS, 265, 996

Canizares, C. R., Davis, J. E., Dewey, D., et al. 2005, PASP, 117, 1144

Crenshaw, D. M., Kraemer, S. B., Boggess, A., et al. 1999, ApJ, 516,750

Czerny, B., Różańska, A., Dovčiak, M., Karas, V., \& Dumont, A.-M. 2004, A\&A, 420, 1

Dauser, T., Garcia, J., Walton, D. J., et al. 2016, arXiv:1601.03771

Dauser, T., García, J., Wilms, J., et al. 2013, MNRAS, 430, 1694

Dauser, T., Wilms, J., Reynolds, C. S., \& Brenneman, L. W. 2010, MNRAS, 409, 1534

de Marco, B., Iwasawa, K., Cappi, M., et al. 2009, A\&A, 507, 159

Done, C., Davis, S. W., Jin, C., Blaes, O., \& Ward, M. 2012, MNRAS, 420, 1848

Dovčiak, M., Bianchi, S., Guainazzi, M., Karas, V., \& Matt, G. 2004a, MNRAS, 350, 745

Dovčiak, M., Karas, V., \& Yaqoob, T. 2004b, ApJS, 153, 205

Emmanoulopoulos, D., Papadakis, I. E., Dovčiak, M., \& McHardy, I. M. 2014, MNRAS, 439, 3931

Galeev, A. A., Rosner, R., \& Vaiana, G. S. 1979, ApJ, 229, 318

Gammie, C. F. 1998, MNRAS, 297, 929

García, J., Dauser, T., Lohfink, A., et al. 2014, ApJ, 782, 76

George, I. M., \& Fabian, A. C. 1991, MNRAS, 249, 352

Goosmann, R. W., Mouchet, M., Czerny, B., et al. 2007, A\&A, 475,155

Haardt, F., \& Maraschi, L. 1993, ApJ, 413, 507
Haardt, F., Maraschi, L., \& Ghisellini, G. 1994, ApJL, 432, L95 Hinshaw, G., Larson, D., Komatsu, E., et al. 2013, ApJS, 208, 19 Ho, L. C., \& Kim, M. 2015, ApJ, 809, 123

Hunter, J. D. 2007, Computing in Science and Engineering, 9, 90 Inoue, H., \& Matsumoto, C. 2003, PASJ, 55, 625

Ives, J. C., Sanford, P. W., \& Penston, M. V. 1976, ApJL, 207, L159

Iwasawa, K., Miniutti, G., \& Fabian, A. C. 2004, MNRAS, 355, 1073

Iwasawa, K., \& Taniguchi, Y. 1993, ApJL, 413, L15

Kallman, T. R., Palmeri, P., Bautista, M. A., Mendoza, C., \& Krolik, J. H. 2004, ApJS, 155, 675

Koyama, K., Tsunemi, H., Dotani, T., et al. 2007, PASJ, 59, 23

Laor, A. 1991, ApJ, 376, 90

Liddle, A. R. 2004, MNRAS, 351, L49

Lightman, A. P., \& Eardley, D. M. 1974, ApJL, 187, L1

Marinucci, A., Matt, G., Miniutti, G., et al. 2014, ApJ, 787, 83

Marziani, P., Sulentic, J. W., Zamanov, R., et al. 2003, ApJS, 145,199

Matt, G., Marinucci, A., Guainazzi, M., et al. 2014, MNRAS, 439, 3016

Matt, G., Perola, G. C., \& Piro, L. 1991, A\&A, 247, 25

Merloni, A., \& Fabian, A. C. 2001, MNRAS, 321, 549

Miller, J. M. 2007, ARA\&A, 45, 441

Miller, L., \& Turner, T. J. 2013, ApJL, 773, L5

Miniutti, G., \& Fabian, A. C. 2004, MNRAS, 349, 1435

Miniutti, G., \& Fabian, A. C. 2006, MNRAS, 366, 115

Molendi, S., Bianchi, S., \& Matt, G. 2003, MNRAS, 343, L1 
Mosquera, A. M., Kochanek, C. S., Chen, B., et al. 2013, ApJ, 769,53

Mushotzky, R. F., Marshall, F. E., Boldt, E. A., Holt, S. S., \& Serlemitsos, P. J. 1980, ApJ, 235, 377

Nandra, K., Barret, D., Barcons, X., et al. 2013, arXiv:1306.2307

Nardini, E., Fabian, A. C., Reis, R. C., \& Walton, D. J. 2011, MNRAS, 410, 1251

Nardini, E., Fabian, A. C., \& Walton, D. J. 2012, MNRAS, 423, 3299

Nayakshin, S., \& Kazanas, D. 2001, ApJL, 553, L141

Niedźwiecki, A., Zdziarski, A. A., \& Szanecki, M. 2016, ApJL, 821, L1

Nordgren, T. E., Helou, G., Chengalur, J. N., Terzian, Y., \& Khachikian, E. 1995, ApJS, 99, 461

Osterbrock, D. E., \& Phillips, M. M. 1977, PASP, 89, 251

Patrick, A. R., Reeves, J. N., Porquet, D., et al. 2011, MNRAS, 411, 2353

Peterson, B. M., Ferrarese, L., Gilbert, K. M., et al. 2004, ApJ, 613, 682

Petrucci, P. O., Ponti, G., Matt, G., et al. 2007, A\&A, 470, 889

Porquet, D., Reeves, J. N., Uttley, P., \& Turner, T. J. 2004, A\&A, 427, 101

Poutanen, J., \& Fabian, A. C. 1999, MNRAS, 306, L31

Reeves, J., Porquet, D., Braito, V., et al. 2016, ApJ, in press (arXiv:1607.01062)

Risaliti, G., Harrison, F. A., Madsen, K. K., et al. 2013, Nature, 494, 449

Risaliti, G., Miniutti, G., Elvis, M., et al. 2009, ApJ, 696, 160

Ross, R. R., \& Fabian, A. C. 1993, MNRAS, 261, 74

Schwarz, G. 1978, Annals of Statistics, 6, 461
Shakura, N. I., \& Sunyaev, R. A. 1973, A\&A, 24, 337

Shakura, N. I., \& Sunyaev, R. A. 1976, MNRAS, 175, 613

Shu, X. W., Yaqoob, T., \& Wang, J. X. 2010, ApJS, 187, 581

Strüder, L., Briel, U., Dennerl, K., et al. 2001, A\&A, 365, L18

Svensson, R., \& Zdziarski, A. A. 1994, ApJ, 436, 599

Tombesi, F., de Marco, B., Iwasawa, K., et al. 2007, A\&A, 467, 1057

Tsujimoto, M., Guainazzi, M., Plucinsky, P. P., et al. 2011, A\&A, 525, A25

Turner, T. J., Miller, L., George, I. M., \& Reeves, J. N. 2006, A\&A, 445, 59

Turner, T. J., Mushotzky, R. F., Yaqoob, T., et al. 2002, ApJL, 574, L123

Uttley, P., McHardy, I. M., \& Vaughan, S. 2005, MNRAS, 359, 345

Vasudevan, R. V., Mushotzky, R. F., Reynolds, C. S., et al. 2014, ApJ, 785, 30

Vaughan, S., Fabian, A. C., Ballantyne, D. R., et al. 2004, MNRAS, 351, 193

Vaughan, S., \& Uttley, P. 2008, MNRAS, 390, 421

Walton, D. J., Nardini, E., Fabian, A. C., Gallo, L. C., \& Reis, R. C. 2013, MNRAS, 428, 2901

Wandel, A., Peterson, B. M., \& Malkan, M. A. 1999, ApJ, 526, 579

Wilkins, D. R., \& Fabian, A. C. 2012, MNRAS, 424, 1284

Wilkins, D. R., \& Gallo, L. C. 2015, MNRAS, 448, 703

Yaqoob, T., \& Murphy, K. D. 2011, MNRAS, 412, 277 